\title{
Methodology for global geodetic time series estimation: A new tool for geodynamics
}

\author{
Philip Davies \\ Ordnance Survey, Southampton, England, United Kingdom. \\ Geoffrey Blewitt ${ }^{1}$ \\ Nevada Bureau of Mines and Geology and Seismological Laboratory, University of Nevada, Reno
}

\begin{abstract}
A method of automatically combining geodetic network solutions to produce station coordinate time series with realistic computed errors has been developed and tested and is being applied on a weekly basis to Global Positioning System (GPS) global and regional networks of the International GPS Service. Our techniques include modified Helmert blocking, stochastic modeling to minimize frame bias, Monte Carlo simulation, variance component estimation, and multiparameter data snooping. An 18-month time series evaluation of 150 globally distributed stations demonstrates that our combined weekly solution is more complete, precise, and reliable than any contributing solution. Our method of attaching regional networks without perturbing the global network solution, rather than combining normal equations, improves the quality measures. The median RMS of station position residuals with respect to a constant velocity model is $2.4 \mathrm{~mm}$ in latitude, $3.0 \mathrm{~mm}$ in longitude, and $7.2 \mathrm{~mm}$ in height. Our solution has since been incorporated into the reference frame ITRF96 (Intemational Terrestrial Reference Frame 1996), showing a RMS coordinate difference of $5.4 \mathrm{~mm}$, the lowest of all contributing solutions. As an independent test, the RMS difference with the ITRF94 is $4.5 \mathrm{~mm}$ in horizontal and $8.1 \mathrm{~mm}$ in height. As a second external test, the station velocity solution was used to estimate plate tectonic Euler vectors, which were then compared with the NUVEL-1A model and found to differ at a level consistent with the computed errors. Given a few more years of data, our error model predicts solutions that will be sufficiently precise to rigorously test NUVEL-1A or its successors.
\end{abstract}

\section{Introduction}

Time series of geodetic network displacements derived from Global Positioning System (GPS) data have been used to investigate geophysical phenomena over distance scales of 1 $10,000 \mathrm{~km}$. Applications have included glaciology, hydrology, volcanology, seismology, tectonophysics, crustal loading, glacial rebound, Earth rotation, and global mass redistribution [Segall and Davis, 1997]. Understandably, networks have typically been implemented and analyzed specifically on a scale and density appropriate to the geophysical signals under investigation [Blewitt, 1993]. We present a methodology to produce a time series of solutions for a unified global network, which, in principle, can accommodate networks at all scales. A longer-term goal of this research is to enable the future production of "densified global solutions" which can be used to investigate signals over all distance scales using a single self-consistent kinematic model. Apart from enabling the study of phenomena without artificial preselection of scale, the generation of such densified global solutions is likely to increase the reliability of geodetic solutions and associated covariance matrices, and it will

\footnotetext{
'Also at Department of Geomatics, University of Newcastle-uponTyne, Newcastle-upon-Tyne, England, United Kingdom.

Copyright 2000 by the American Geophysical Union.
}

Paper number 2000JB900004.

0148-0227/00/2000JB900004\$09.00 improve the utility and consistency of archived solutions for future generations of Earth scientists.

This contribution aims to present the geodetic methodology and demonstrate its potential as a new tool for geodynamics. It begins by explaining the method in some detail thus providing a suitable reference for further work that might utilize this method or for researchers who use our products and need a more definitive understanding of them. The method has been tested on an 18-month subset of data from the International GPS Service (IGS) global and regional networks (see http://igscb.jpl.nasa gov/). The results demonstrate a high level of precision and reliability; the median RMS of three-dimensional station position residuals for 1-week solutions with respect to a linear motion model is shown to be $<5 \mathrm{~mm}$.

Since our coordinate solution was subsequently submitted to the International Earth Rotation Service (IERS) for incorporation into ITRF96 (International Terrestrial Reference Frame 1996), this paper describes preliminary assessment in comparison with ITRF94, a completely independent solution. IERS has published ('http://lareg.ensg.ign.fr/ITRF/ITRF96-rep.html\#results') their independent assessment of precision, showing our solution to have a coordinate RMS of $5.4 \mathrm{~mm}$ with respect to the final realization of ITRF96. According to IERS this is the smallest RMS of any contributing solution.

Finally, to demonstrate the method's potential as a geophysical research tool, preliminary investigations were carried out on the estimation of global-scale geophysical parameters, including Euler vectors for tectonic plates. We conclude that geodetic resolution of plate kinematics should be sufficient to 
rigorously test geological models such as NUVEL-1 A [DeMets et al., 1994] within the next few years [Larson et al., 1997].

\section{Methodology: Network Combination}

\subsection{Geodetic Background}

We follow Mueller [1985] in using "Terrestrial Reference System" (TRS) to mean the conventional theoretical definition of the coordinate basis (i.e., the geodetic datum) and using "Terrestrial Reference Frame" (TRF) to mean a particular realworld implementation of the reference system by stating coordinates (positions and sometimes velocities) of ground marks. In practice, accurate models of surface kinematics (e.g., due to tidal effects) are introduced into the TRS and are therefore embedded into the definition of coordinates. A TRS is essentially arbitrary and conveniently defined; a TRF is estimated through physical observation and hence can only be stated in statistical terms. Therefore kinematics must be interpreted within the context of both the TRS definition and the TRF statistics.

Various investigators have developed methods to estimate a free network TRF using a global network of permanent GPS stations (known as a polyhedron network), without the use of a priori information. [Heflin et al., 1992; Blewitt et al., 1992; and Herring et al., 1993]. Not only can we estimate the internal geometry of the network polyhedron, but also the evolution (with respect to the TRF) of the Earth rotation vector (polar motion) [Lindqwister et al., 1992], and the Earth center of mass (geocenter) [Vigue et al., 1992]. The evolution of the geocenter and the overall network scale are useful indicators of systematic error (e.g., orbit dynamics, atmospheric delay, and antenna effects). Methods for estimating free network TRF kinematics from GPS data were soon thereafter demonstrated and applied to crustal deformation [Blewitt et al., 1993b; and Bock et al., 1993], and atmospheric loading [van Dam et al., 1994], and global-scale plate tectonics [Argus and Heflin, 1995].

\subsection{IGS Densification Pilot Project}

The IGS provides GPS orbits, tracking data, and other GPS data products to meet the objectives of a wide range of scientific and engineering applications and studies. Our research has been carried out in the context of the IGS densification pilot project, initiated in September 1995 [Blewitt et al., 1993a; Beutler et al., 1995; Zumberge and Liu, 1995]. One of the objectives of this project was to produce a unified and consistent station coordinate solution on a weekly basis [Blewitt et al., 1995]. The success of this pilot project has led to the start of the fully operational phase in January 1999.

While the emphasis of this paper is on the improvement in solution quality that results from network combination methodology, network combination was initially seen as a practical necessity to overcome the immense computational burden of processing the entire IGS network containing hundreds of stations. A distributed approach to estimating the weekly polyhedron was therefore developed. The agencies contributing analysis results to the project are listed in Table 1. The jargon terms, which are used in IGS to describe the various network solutions, are summarized in Table 2. Figure 1 explains the distributed processing approach schematically. The following steps summarize this scheme:

1. Each IGS Analysis Center (AC) produces a global coordinate solution known as an "A network" every week. These solutions contain parameter vectors, a full covariance matrix, and a full a priori covariance matrix. The latter allows us to remove a priori constraints to produce a free network solution.

2. Each IGS Global Network Associate Analysis Center (GNAAC) produces a combined A network solution, known as a "G network", every week. To exploit redundancy for improved quality, only "global" stations may be included in the G network. A global station is defined as a station appearing in at least three AC solutions in the week in question; hence there are a few nonglobal stations in most $\mathrm{AC}$ solutions, and the total number of

Table 1. IGS Agencies participating in the IGS Densification Pilot Project Since Before February 1997

\begin{tabular}{|c|c|c|c|}
\hline $\operatorname{Code}^{a}$ & Type $^{b}$ & Agency Name & Location \\
\hline COD & $\mathrm{AC}$ & Center for Orbit Determination in Europe & Berne, Switzerland \\
\hline EMR & $\mathrm{AC}$ & Natural Resources Canada, Geodetic Surveys & Ottawa, Ontario, Canada \\
\hline ESA & $\mathrm{AC}$ & European Space Agency & Darmstadt, Germany \\
\hline GFZ & $\mathrm{AC}$ & GeoForschungsZentrum & Potsdam, Germany \\
\hline JPL & $\mathrm{AC}$ & Jet Propulsion Laboratory & Pasadena, California, United States \\
\hline NGS & $\mathrm{AC}$ & $\begin{array}{l}\text { National Oceanic and Atmospheric } \\
\text { Administration }\end{array}$ & Silver Spring, Maryland, United States \\
\hline SIO & $\mathrm{AC}$ & Scripps Institute of Oceanography & San Diego, California, United States \\
\hline EUR & RNAAC & European regional net from COD & Berne, Switzerland \\
\hline GIA & RNAAC & University of Alaska Geophysical Institute & Fairbanks, Alaska, United States \\
\hline GSI & RNAAC & Geographical Survey Institute & Tsukuba, Japan \\
\hline PGC & RNAAC & Pacific Geoscience Center & Victoria, British Columbia, Canada \\
\hline SIR & RNAAC & Deutsches Geodaetisches Forschungsinstitut & München, Germany \\
\hline JPL & GNAAC & Jet Propulsion Laboratory & Pasadena, California, United States \\
\hline MIT & GNAAC & Massachusetts Institute of Technology & Cambridge, Massachusetts, United States \\
\hline NCL & GNAAC & University of Newcastle upon Tyne & Newcastle, England, United Kingdom \\
\hline
\end{tabular}

These code names are official International GPS Service designations used in file names and data products.

'Abbreviations are as follows: AC, Analysis Center; RNAAC, Regional Network Associate Analysis Center; GNAAC, Global Network Associate Analysis Center; GPS, Global Positioning System. 
Table 2. Definition of Terms

\begin{tabular}{|c|c|c|}
\hline Network Type & Producing Centre Type & Description \\
\hline A network & Analysis Center (AC) & fundamental global network solution containing mostly global stations \\
\hline R network & $\begin{array}{l}\text { Regional Network Associate } \\
\text { Analysis Center (RNAAC) }\end{array}$ & $\begin{array}{l}\text { regional network solution with orbit constraints; contains the regional } \\
\text { stations of a particular region plus at least three global stations in } \\
\text { that region }\end{array}$ \\
\hline G network & $\begin{array}{l}\text { Global Network Associate } \\
\text { Analysis Center (GNAAC) }\end{array}$ & combination of global station estimates in A networks \\
\hline P network & GNAAC & $\begin{array}{l}\text { complete polyhedron including } G \text { network with } R \text { networks and } \\
\text { nonglobal A network stations attached }\end{array}$ \\
\hline
\end{tabular}

global stations varies from week to week. Note that this specific sense of the term "global" is intended throughout this paper.

3. Each IGS Regional Network Associate Analysis Center (RNAAC) produces a regional coordinate solution known as an " $R$ network" every week. The analysis performed by the RNAACs use the official IGS orbits, which have been produced by combining $\mathrm{AC}$ orbit solutions and aligning the orbits to ITRF. To enable network combination in the next step, each $R$ network must include at least three global stations. All nonglobal stations are called "regional" stations. Note that our GNAAC also produces a pseudo $\mathrm{R}$ network, using stations that did not count as global stations in step 2.

4. Each GNAAC then produces a complete IGS polyhedron network, known as the "P network," by combining the $G$ network with the R networks every week. The use of IGS orbits by the RNAACs means that the R networks are oriented to ITRF, so removing a priori constraints is not as straightforward as for $\mathrm{A}$ networks. We address this problem through the stochastic model (see section 2.4). As will also be explained, we have developed a method of combination which is better described as an "attachment," where the G network is not allowed to be perturbed by the $\mathrm{R}$ networks. Therefore the stations in common between the $G$ network and final $P$ network have identical coordinates and covariances.

5. The resulting $G$ networks or $P$ networks are available to geophysicists who require more advanced global geodetic control (e.g., for tide gauge benchmark monitoring). One objective of this paper is to help users to have a better understanding of these products.

6. The resulting weekly $\mathrm{G}$ networks or $\mathrm{P}$ networks are further combined into a kinematic model, which is then submitted as an input into the next realization of ITRF. This methodology is

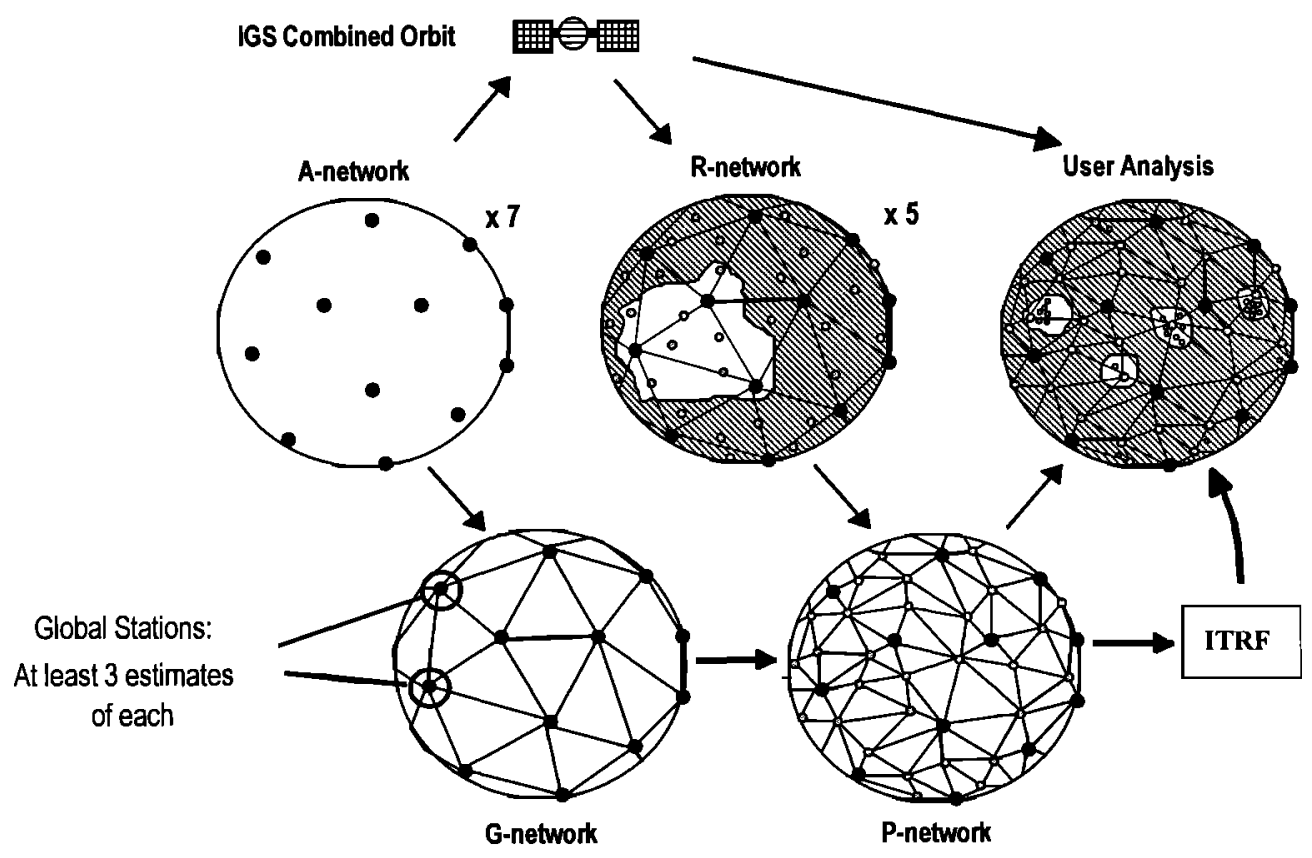

Figure 1. The distributed polyhedron assembly scheme of the International GPS Service, showing the four types of network solution. A networks are produced by seven Analysis Centers (Table 1), which also produce orbit solutions, which are combined to produce the IGS combined orbit. R Networks are produced by four Regional Network Associate Analysis Centers (Table 1), using the combined orbit and at least three global stations. Our Global Network Associate Analysis Center analysis produces the $\mathrm{G}$ network and $\mathrm{P}$ network. A kinematıc model is fit to the $\mathrm{P}$ network to produce the $\mathrm{kP}$ solution, which contributes to International Terrestrial Reference Frame realizations. 
described and tested in this paper. The specific solution described here was subsequertly included in ITRF96. This is an indirect and more conventional route for users to access our products.

The geodetic solutions are represented in SINEX (Software Independent Exchange) format, which was developed to facilitate the pilot project (but has since been adopted by other space geodetic techniques). One advantage of the SINEX format is that station information is documented, and hence the solutions can be corrected after the fact should this information require correction. This is especially important for correcting antenna heights and phase center offsets.

Although the procedure above was developed as a general scheme [Blewitt et al., 1993a, 1995], our GNAAC methodology incorporates specific techniques, which will be discussed. Our techniques include modified Helmert blocking, stochastıc modeling to minimize frame bias, Monte Carlo simulation, variance component estimation, and multıparameter outlier detection.

\subsection{Network Combination}

The aim of this contribution is to explore the potential for improving the precision and accuracy of station coordinates and kinematics through network combination analysis, which brings increased spatial coverage, redundancy, and the possibility for improved reliability through quality analysis techniques, including automatic outlier detection and variance component analysis. Methods for comparison and combination of geodetic network coordinate solutions have been introduced and developed by Kosters [1988], Kosters and Kok [1989], Boucher and Altamimı [1993]. This is an unusual least squares task in two respects. First, the input data types are the same as the output parameter types (that is, a 3-D position of each station at a stated epoch and optionally a constant 3-D velocity of each station). Second, the covariance matrices of the blocks of input data are fully populated (because each block is the output parameter set of an earlier least squares analysis).

In our approach the reference system (datum) definition is dealt with via the stochastic model so our central functional model involves a simple reorderıng of parameters. Consider an input parameter list $\mathrm{x}$ ( $m$ entries) of a parameter vector $\mathbf{x}$ with covariance matrix $\Sigma_{\mathbf{x}}$, which is to be related to another parameter list y ( $n$ entries). For notational convenience the matrix

$$
\underset{x-y}{\mathbf{A}}(n \times m)
$$

is defined as the left-multiplying linear operator which reparameterizes $\mathbf{x}$ and $\Sigma_{\mathbf{x}}$ from list $\mathbf{x}$ to list $\mathbf{y}$. The elements of this matrix are zero except for elements $a_{i j}=1$, where member $i$ of parameter list $\mathrm{y}$ is identified as the same parameter as member $j$ of list $\mathbf{x}$. Using overbars for the reparameterized arrays,

$$
\overline{\mathbf{x}}=\underset{\mathbf{x}-\mathrm{y}}{\mathbf{A}} \mathbf{x} \text { and } \Sigma_{\overline{\mathbf{x}}}=\underset{\mathbf{x}-\mathbf{y}}{\mathbf{A}} \Sigma_{\mathbf{x}} \underset{\mathrm{x}-\mathrm{y}}{\mathbf{A}^{\prime}}
$$

where $\Sigma_{\mathbf{x}}$ is an associated covariance, inverse covariance, or information matrix. A has the properties

$$
\underset{y-x}{\mathbf{A}}=\underset{\mathbf{x}-\mathbf{y}}{\mathbf{A}^{\prime}} \text { and } \underset{z-\mathbf{x}}{\mathbf{A}}=\underset{\mathbf{y}-\mathbf{x} z-\mathbf{y}}{\mathbf{A}} \text {. }
$$

From each input SINEX solution we can extract the estimate parameter vector $\mathbf{x}$ and its covariance matrix $\Sigma_{\mathbf{x}}$, the a priori parameter vector $\mathbf{z}$, and its covariance matrix $\Sigma_{\mathbf{z}}$. It is then straightforward to use a station catalogue (again, in SINEX format) to compute

$$
\underset{\mathbf{x}-\mathbf{c}}{\mathbf{A}} \text { and } \underset{\mathbf{z}-\mathbf{c}}{\mathbf{A}}
$$

between the estimation parameter list $\mathrm{x}$, the a priori parameter list $\mathrm{z}$, and the catalogue parameter list $\mathrm{c}$.

\subsection{Removing A Priori Constraints}

All nonminimal constraints on station coordinates stated in the SINEX file in the form of an a priori parameter vector and covariance matrix were removed using the principle of removal and addition of information from a normal equation system:

$$
\begin{gathered}
\Sigma_{\overline{\mathbf{x}}}=\left(\Sigma_{\mathbf{x}}^{-1}-\underset{\mathbf{z}-\mathbf{x}}{\mathbf{A}} \Sigma_{\mathbf{z}}^{-1} \underset{\mathbf{x}-\mathbf{z}}{\mathbf{A}}+\left(\mathbf{C}^{\prime} \mathbf{C}\right)^{-1} \mathbf{C}^{\prime} \Sigma_{\mathbf{w}}^{-1} \mathbf{C}\left(\mathbf{C}^{\prime} \mathbf{C}\right)^{-1}\right)^{-1}, \\
\overline{\mathbf{x}}=\Sigma_{\overline{\mathbf{x}}}\left(\Sigma_{\mathbf{x}}^{-1} \mathbf{x}-\underset{\mathbf{z}-\mathbf{x}}{\mathbf{A}} \Sigma_{\mathbf{z}}^{-1} \mathbf{z}\right),
\end{gathered}
$$

where the overbar denotes the resulting deconstrained solution. The third term on the right-hand side of (1) is required for A networks that exhibited near-singular normal equations when stated constraints were removed. The term $\left(\mathbf{C}^{\prime} \mathbf{C}\right)^{-1} \mathbf{C}^{\prime}$ is a generalized inverse of the linearized functional matrix $\mathbf{C}$ of three loose constraints $w$ of 3 -D network orientation, that is, the first three rows of (A3).

The elements of the diagonal covariance matrix $\Sigma_{w}$ are chosen in each case to give good matrix conditioning while not being too tight to cause numerical error. Because these extra constraints are minimal, they have no effect on the deconstrained parameters in (2).

The A networks sense the geocentric origin and network scale, and this information was retained in the combined $G$ network. Any orientation information in these networks is due to artificial constraints and was removed to prevent network distortion. In the case of $\mathrm{R}$ networks the ability to sense the geocenter and network scale depends on network size. Small R networks can be assumed to be insensitive to the geocenter, while large ones have some sensitivity. We assumed that no $\mathrm{R}$ network provides information of value on the origin or network scale; the $G$ network origin is defined by the combination of A networks alone. Therefore in the $\mathrm{R}$ networks the linear combinations of parameters corresponding to all seven Helmert parameters (3-D origin, 3-D orientation and scale) were therefore assumed to be uninformative. This is reasonable because the reference system definition of the regional networks (especially small ones) comes largely from the IGS combined orbits, which, in turn, are positioned in the ITRF. The combined G network does not require this a priori information.

Our approach is to effectıvely remove the artificial reference system definition by augmenting the deconstrained estimate covariance matrix such that the standard errors of the unobserved Helmert parameters become large. This is a loose minimal constraints approach which avoids introducing reference system parameters into the network combination functional model. The linear combinations of coordinates corresponding to the Helmert parameters are the rows of $\mathbf{B}_{\mathbf{X}}$ in (A3). The covariance matrix of the deconstrained parameters is augmented to remove Helmert parameter constraints by either:

$$
\begin{gathered}
\Sigma_{\overline{\mathbf{x}}}=\Sigma_{\overline{\mathbf{x}}}+\mathbf{C}^{\prime} \Sigma_{\mathbf{w}} \mathbf{C}, \text { or } \\
\Sigma_{\overline{\mathbf{x}}}=\left(\Sigma_{\overline{\bar{x}}}^{-1}-\Sigma_{\overline{\mathbf{x}}}^{-1} \mathbf{C}^{\prime}\left(\mathbf{C} \Sigma_{\overline{\mathbf{x}}}^{-1} \mathbf{C}^{\prime}+\Sigma_{\mathbf{w}}\right)^{-1} \mathbf{C} \Sigma_{\overline{\mathbf{x}}}^{-1}\right)^{-1},
\end{gathered}
$$


where the curly overbar denotes the augmented deconstrained matrix. C is made up of the appropriate rows of $\mathbf{B}$ for the reference system parameters that are to be augmented (three orientations in the case of A networks, and all seven parameters in the case of R networks). $\Sigma_{\mathrm{w}}$ is the diagonal covariance matrix of the augmented parameters. In the terminology of Blewtt [1998] this is a "loosening transformation," where ( 3 ) is the covariance augmentation formulation, and (4) is the weight reduction formulation. Although the formulations are equivalent, each has its advantages depending on the computation involved. From the computation of (1), $\Sigma_{\bar{x}}$ and $\Sigma_{\overline{\mathbf{x}}}^{-1}$ are both known, so by using both (3) and (4), $\Sigma_{\widetilde{\mathbf{x}}}$ and $\Sigma_{\tilde{\mathbf{x}}}^{-1}$ can both be computed without a further matrix inversion. Because the information being removed here is extrinsic to the network (i.e. the function C is orthogonal to the network observations), no parameter update is required.

In A networks we find a wide range of stated and unstated constraints. Stated station constraints can be loose $(10 \mathrm{~m}$ standard error) or tight (1 $\mathrm{mm}$ standard error). On removing these constraints from A networks, we would expect the deconstrained normal equations to have a rank deficiency of three (computationally, three very small eigenvalues) corresponding to the three degrees of freedom of the unobserved network orientation. However, some A networks include unstated constraints that make the normal equations quite regular with constraints removed, while others have the singular normals we would expect. These differences come from the different methods employed in network estimation software. Some A networks had unstated constraints only of $\mathrm{X}$ and $\mathrm{Y}$ orientation, not of $Z$, or vice versa. All these cases of stated and unstated constraint were handled by the deconstraint procedure. We must assume that $A$ network unstated constraints are minımal (i.e. of orientation only); nonminimal unstated constraint cannot be distinguished from observation information. As long as this is the case, (1) and (2) are still valid (i.e., we can obtain the undistorted network except for certain known linear combinations corresponding to the equations of global orientation, which can be dealt with).

\subsection{Modified Helmert Blocking Procedure}

The combination of A networks (to form the $G$ network) and subsequent attachment of $\mathrm{R}$ networks (to form the $P$ network) is accomplished by a procedure similar to Helmert blocking but with important modifications we have developed. Wolf [1978] summarizes the history of the Helmert blocking method. The modifications are that (1) only A networks (not $\mathrm{R}$ networks) contribute to the estimation of "junction" stations that are in common to both an A network and R network and (2) only stations estimated by at least three A networks are allowed in the $\mathrm{G}$ network. This gives the combined $\mathrm{G}$ network the redundancy required to carry out variance component estimation and outlier detection, which enables a high-reliability primary reference frame.

Because observations and parameters are the same quantity types (coordinates), the lınear observation model is exact, making nominal parameter values unnecessary. There are $n$ input components (A and $\mathrm{R}$ networks); for each component $i$ we have observations $\mathbf{x}_{i}$ with covariance submatrix $\Sigma_{\mathbf{x}_{\text {, }}}$ and parameter list $x_{l}$. Let the common (junction) parameter list be $g$. The local parameter list $\mathrm{u}_{i}$ for each block contains $\left\{\mathrm{u}_{i}\right\}=\left\{\mathrm{x}_{i}\right\} \notin\{\mathrm{g}\}$. We estimate the common parameters $g$ and when required the block- by-block local parameters $\mathbf{u}_{i}$. The overall normal equation system for this estimation can be written as a stack of components, one for each $\mathrm{G}$ network:

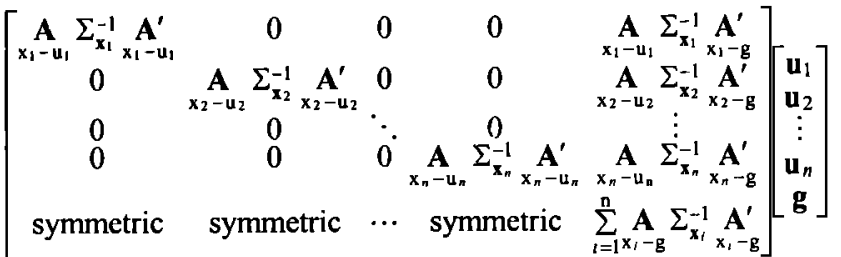

$$
\begin{aligned}
& =\left[\begin{array}{cc}
\underset{\mathbf{x}_{1}-u_{1}}{\mathbf{A}} & \Sigma_{\mathbf{x}_{1}}^{-1} \mathbf{x}_{1} \\
\underset{\mathbf{x}_{2}-\mathbf{u}_{2}}{\mathbf{A}} & \Sigma_{\mathbf{x}_{2}}^{-1} \mathbf{x}_{2} \\
\underset{\mathbf{x}_{n}-u_{n}}{\mathbf{A}} & \vdots \\
\sum_{l=1}^{n}\left(\underset{\mathbf{x}_{n}}{-1} \mathbf{x}_{n}-\mathbf{g}\right. & \left.\Sigma_{\mathbf{x}_{l}}^{-1} \mathbf{x}_{l}\right)
\end{array}\right] .
\end{aligned}
$$

Cooper [1987] shows that to efficiently solve such a sparse system, we can use

$$
\Sigma_{\mathrm{g}}=\left(\sum_{i=1}^{n} \sigma_{i}^{-2} \mathbf{N}_{t}\right)^{-1} \quad \mathbf{g}=\Sigma_{\mathrm{g}} \sum_{l=1}^{n} \sigma_{i}^{-2} \mathbf{d}_{t},
$$

with residuals

$$
\mathbf{v}_{\iota}=\underset{\mathbf{g}-\mathbf{x}_{i}}{\mathbf{A}} \mathbf{g}-\mathbf{x}_{t} \quad \Sigma_{\mathbf{v}_{i}}=\Sigma_{\mathbf{x}_{t}}-\underset{\mathbf{g}-\mathbf{x}_{i}}{\mathbf{A}} \Sigma_{\mathbf{g}} \underset{\mathbf{g}-\mathbf{x}_{i}}{\mathbf{A}^{\prime}}
$$

where

$$
\begin{aligned}
& \mathbf{N}_{i}=\mathbf{E}_{l}-\mathbf{D}_{i}^{\prime} \mathbf{C}_{t}^{-1} \mathbf{D}_{i}, \quad \mathbf{d}_{i}=\mathbf{b}_{i}-\mathbf{D}_{l}^{\prime} \mathbf{C}_{l}^{-1} \mathbf{a}_{l} \\
& \mathbf{a}_{i} \equiv \underset{\mathbf{x}_{i}-\mathbf{u}_{i}}{\mathbf{A}} \Sigma_{\mathbf{x}_{i}}^{-1} \mathbf{x}_{i}, \quad \mathbf{b}_{i} \equiv \underset{\mathbf{x}_{i}-\mathrm{g}}{\mathbf{A}} \Sigma_{\mathbf{x}_{i}}^{-1} \mathbf{x}_{i} \\
& \mathbf{C}_{i} \equiv \underset{\mathbf{x}_{i}-\mathbf{u}_{t}}{\mathbf{A}} \Sigma_{\mathbf{x}_{i}}^{-1} \underset{\mathbf{x}_{i}-\mathbf{u}_{i}}{\mathbf{A}^{\prime}}, \quad \mathbf{D}_{l} \equiv \underset{\mathbf{x}_{i}-\mathbf{u}_{i}}{\mathbf{A}} \Sigma_{\mathbf{x}_{i}}^{-1} \mathbf{x}_{\mathbf{x}_{i}-\mathrm{g}}^{\mathbf{A}^{\prime}},
\end{aligned}
$$

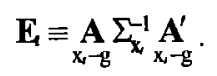

Here $\mathbf{N}_{\imath}$ and $\mathbf{d}_{\text {, }}$ are the reduced normal equation components for block $i$. The factors $\sigma_{i}^{2}$ applied in these equations are block variance components discussed below. 'The local parameters and their covariance submatrices are subsequently obtained when required by

$$
\mathbf{u}_{\imath}=\mathbf{C}_{l}^{-1} \mathbf{a}_{l}-\mathbf{C}_{l}^{-1} \mathbf{D}_{\imath} \mathbf{g} \quad \Sigma_{\mathbf{u}_{t}}=\mathbf{C}_{l}^{-1}+\mathbf{C}_{i}^{-1} \mathbf{D}_{\imath} \Sigma_{\mathbf{g}} \mathbf{D}_{l}^{\prime} \mathbf{C}_{l}^{-1},
$$

and the off-diagonal covariance submatrices of the solution are

$$
\Sigma_{\mathbf{u}, \mathbf{g}}=-\mathbf{C}_{i}^{-1} \mathbf{D}_{\imath} \Sigma_{\mathbf{g}} \quad \Sigma_{\mathbf{u}, \mathbf{u}_{\mathfrak{l}}}=\mathbf{C}_{i}^{-1} \mathbf{D}_{\boldsymbol{i}} \Sigma_{\mathbf{g}} \mathbf{D}_{j}^{\prime} \mathbf{C}_{j}^{-1} .
$$

For every weekly epoch we obtain loose global A network solutions $\left(\mathbf{a}_{1}, \Sigma_{\mathbf{a}_{i}}\right)$ and similarly loose regional R networks ( $\mathbf{r}_{i}$, $\Sigma_{r_{i}}$ ). The set of Helmert blocks $x_{i}$ includes $a_{i}$ and $r_{i}$. The loose $G$ and $P$ networks are estimated from the loose $A$ and $R$ networks as follows:

1. A global parameter list $g$ is written which includes stations estimated by three or more A networks. The local parameter list $u_{i}$ for each A network includes all the stations in that network not in $\mathrm{g}$. The stackıng procedure of (6) is used to give the $G$ network estimate (i.e. the common parameters $\mathbf{g}$ of the Helmert 
blocking procedure) using only the A network contributions to the summation, that is, each $\mathbf{x}_{\boldsymbol{t}} \equiv \mathbf{a}_{\mathbf{r}}$

2. Each $R$ network (regional solution) is attached to the $G$ network by means of the three or more G-stations included in the $R$ network as junction stations. This is done by back substitution of the $G$ network estimates of the junction stations into the $R$ network by (5)-(9) where each $\mathbf{x}_{i} \equiv \mathbf{r}_{i}$. Hence the global stations of the resulting combined $P$ network are unaffected by the $R$ networks.

3. The back substitution of $R$ networks includes a pseudo $R$ network which is included to deal with the nonglobal A network stations. This is a combination of A networks including only the nonglobal stations and a core set of about 20 well-distributed global stations. The core stations act as the junction stations in attaching this $\mathrm{R}$ network to the $\mathrm{P}$ network in the same way as the real $R$ networks. Any stations which appear in the pseudo $R$ network and a real $\mathrm{R}$ network are deleted from the pseudo $\mathrm{R}$ network.

This procedure follows that of a standard Helmert blocking solution of the $A$ and $R$ networks, except that the common parameter list does not include all the common parameters but only those that meet the global station requirements. Most importantiy, the $\mathrm{R}$ networks do not contribute to the common parameter estimation. The approach can be thought of as equivalent to a simultaneous least squares estimation from all the data in the limit of large variances being ascribed to the $R$ network junction station estimates. The result is that the $G$ network estimate and its covariance matrix are determined only by the A networks, and this forms a first-order control network to which the regional components are attached.

\subsection{Variance Component Estimation}

The scaling factors $\sigma_{t}^{2}$ applied to the AC global networks in (6) are required because the relative scaling of the input $A$ network covariance matrices is not correct. We determined these factors by a damped variance component estimation technique. The scaling factor for each $\mathrm{AC}$ in any week $i$ was that determined in week $i-1$. In week $i$ the factor was updated based on the residuals (7) of each AC global network. We found that the classical Helmert method of computing variance and covariance components [Koch, 1987a; Grafarend and Schaffrin, 1979] is not useful in practice for the following reasons: (1) the full covariance matrix of observations is inverted, which is unnecessary because our observation blocks are uncorrelated; and (2) negative variance components can arise, which are meaningless and prevent convergence of the solution. Instead, we used the following well-behaved nonnegative algorithm (derived from expressions given by Sahin et al. [1992]):

$$
s_{i, j}^{2}=\frac{\hat{\mathbf{v}}_{i}^{\prime} \Sigma_{\mathbf{x}_{i}}^{-1} \hat{\mathbf{v}}_{i}}{m_{1}-\operatorname{tr}\left(\Sigma_{\hat{\mathbf{g}}} \sigma_{i, j}^{-2} \mathbf{N}_{i}\right)}, \quad \sigma_{i, j+1}^{2}=\sigma_{i, j}^{2} s_{i, j}^{2},
$$

where $m_{i}$ is the number of observations in block $i$ and $j$ is the iteration counter. Equation (10) can be understood as a partition of the total sum of squares of the residuals. The numerator is the weighted sum of squares of residuals of the $t$ th observation block, and the denominator is the redundancy number of that observation block.

Equations (6), (7), and (10) could be iterated from $\sigma_{\mathrm{l}}=1$ until $s_{l} \approx 1$. However, because our aim was to determine a slowly evolving scaling factor for each $\mathrm{AC}$ over many weeks of analysis in the context of outlier detection and removal (see section 2.8 below), we chose to perform only one update of variance components in each global network combination and to damp the update by

$$
\sigma_{i, j+1}^{2}=\sigma_{i, j}^{2}\left(d s_{i, j}^{2}-d+1\right),
$$

where the damping factor $d=0.2$. This reduces the effect of outlying observations that can cause variance components to fluctuate wildly; we preferred to deal with these by outlier detection and removal. Setting $d$ too close to unity can render outlier detection impotent (since the outliers distort covariance scaling). In this application where we expect a constant scaling factor for each AC, a highly damped approach is preferable.

\subsection{Monte Carlo Confidence Intervals}

To be statistically rigorous, variance components should only be applied after the null hypothesis (that the functional and stochastic models are correct) has been disproved. This is very difficult to do because the variance component probability distribution is unknown. We derived a $90 \%$ confidence interval by the Monte Carlo method, which could be used to solve this problem, although this was not done routinely in our weekly analysis. Analytic derivations of variance component density functions "are restricted to special applications, or they give only approximate results" [Koch, 1987b]. Bayesian methods have been developed by Koch [1987b, 1988] and $O u$ [1991]; these use numerical integration and approximations.

To determine the variance component confidence intervals by Monte Carlo, we generated 100 sets of pseudorandomly deviating A network coordinates. These coordinate sets were generated such that the mean coordinate values are those of a real set of A networks from a typical week, and the deviations follow the multivariate normal distribution defined by the real $A$ networks' covariance matrices. Equations (6), (7) and (10) were iterated 4 times to ensure convergence of the variance component estımation. The combined global network residuals are then due entirely to the pseudorandom behavior of the simulated observations (i.e. the null hypothesis is fulfilled a priori and we know the true variance components are unity). Table 3 gives the results, including the $90 \%$ confidence interval of each variance component.

\subsection{Iterative Data Snooping}

Two blunder types were repeatedly found in the A and R networks during the pilot project: (1) reduction of the observation point to the monument using an incorrect eccentricity vector (i.e., getting the antenna height wrong); (2) misidentification of the antenna with another GPS station at the same site. Some station antenna heights were poorly reported in this early stage of the IGS project. Following the combined G network estimation, we used the alternative hypothesis

$$
\mathbf{C}=\left[\begin{array}{lll:l:l:l:lll:l:l}
0 & 0 & 0 & & & & & 0 & 0 & & \\
0 & 0 & 0 & 0 & \cdots & 0 & 1 & 0 & \cdots & 0 \\
0 & 0 & 0 & & & 0 & 0 & 1 & &
\end{array}\right]
$$

in (A8) and (A9) to compute marginally detectable errors (MDEs) [Baarda, 1968] and apply an iterative data snooping (outlier testing) to each A network station position estimate (coordinate triplet) in turn against a chi-square distribution with three degrees of freedom (following Kosters and Kok [1989]). C is zero except for the triplet block corresponding to the $i$ th station 
Table 3. Summary of Monte Carlo Variance Component Confidence Intervals

\begin{tabular}{lcccccc}
\multicolumn{1}{c}{ Week 0862 A Networks } & COD & EMR & ESA & GFZ & JPL & SIO \\
\hline Number of stations & 60 & 30 & 52 & 50 & $5 ?$ & 59 \\
A priori scale factor & 49.79 & 13.82 & 22.37 & 34.31 & 30.24 & 2.888 \\
Network degrees of freedom & 124.7 & 79.73 & 146.1 & 109.1 & 124.0 & 124.4 \\
Mean of $\sigma^{2}$ & 1.01 & 1.00 & 1.01 & 1.02 & 0.99 & 1.01 \\
Standard error of $\sigma^{2}$ & 0.15 & 0.15 & 0.12 & 0.19 & 0.13 & 0.14 \\
5th percentile & 0.77 & 0.77 & 0.83 & 0.73 & 0.82 & 0.78 \\
50th percentile & 1.01 & 1.00 & 0.99 & 1.01 & 0.97 & 1.02 \\
95th percentile & 1.24 & 1.20 & 1.22 & 1.36 & 1.21 & 1.21 \\
\hline
\end{tabular}

estimate. The station estimate with the largest $T$ value in (A9) is discarded, and the combined $G$ network estimation and outlier removal step are iterated until the largest $T$ is below the $99.9 \%$ confidence critical value. If any one station is rejected from two A networks in the iterative process, it was entirely discarded from that week's $G$ network. (These stations were those where ACs apply different antenna height reductions; we did not attempt to manually correct these errors). The variance component updates (10) for the following week's analysis were then computed from the final $\mathrm{G}$ network iteration.

\subsection{Monte Carlo Testing of Iterative Data Snooping}

It is not clear what the MDE and noncentrality parameter in (A7) mean in the context of iterative data snooping, because we only test the station estimate with the largest value of $T$ at each iteration, and the input data may have multiple outliers. Iterative data snooping is therefore a practical technique without much theoretical justification. We ran a Monte Carlo test to determine the probability of correct action, type I errors (finding an outlier when none exists), or type II errors (not finding an actual outlier) in the first iteration. As before, for each test 100 sets of pseudorandom data were generated such that the mean parameter values are those of a real set of A networks from a typical week, and the random deviations follow the multivariate normal distribution defined by the real A networks' covariance matrices. We then inserted outliers of size $2 / 3 \mathrm{MDE}, 1 \mathrm{MDE}$, or $3 / 2 \mathrm{MDE}$ in zero, one, or two input estimates of a single station, computed (6) and (7), and carried out the first iteration of the outlier detection test at a confidence level of $99.9 \%$. The results are shown in Table 4.

It is perhaps surprising that with a confidence level of $99.9 \%$, the test will reject an observation one time in four when no outliers or other model errors are present! The iteration then continues, so the test will reject two observations one time in sixteen, etc. This result should be bome in mind by anybody using this type of test blindly. If a single outlier is caught successfully, we return to the no-outliers situation on the second iteration, so the probability of catching one outlier of $1 \mathrm{MDE}$ size and then stopping is 0.56 . Similarly, the probability of catching two outliers of $1 \mathrm{MDE}$ size and then stopping is 0.48 . This gives some insight into what MDE means in practice. Monte-Carlo testing could be extended to many-outlier situations.

\section{Methodology: Network Kinematics and Plate Tectonics}

\subsection{Estimation of Kinematic Network Solutions}

From the time series of weekly polyhedron solutions we estimated a kinematic network solution which includes a reference epoch position and a constant velocity for each station. A four dimensional Terrestrial Reference System has 14 degrees of freedom, including the seven usual Helmert parameters plus their time derivatives. Let the kinematic parameter vector $\mathbf{k}$ be defined as in (A4). The linear model for the epoch $t$ observation block $x_{t}$ is

Table 4. Summary of Monte Carlo Tests of Iterative Outlier Detection

\begin{tabular}{lcccc}
\hline \multirow{2}{*}{$\begin{array}{c}\text { Outlier } \\
\text { Size }\end{array}$} & $\begin{array}{c}\text { Number } \\
\text { of } \\
\end{array}$ & Outliers & Probability of First Iteration Test Decision Being \\
\cline { 3 - 5 } & & Correct & Type II Error Only & Type I and II Error \\
\hline None & 0 & 0.75 & 0 & $0.25^{\mathrm{a}}$ \\
2/3 MDE & 1 & 0.23 & 0.59 & 0.19 \\
$1 \mathrm{MDE}$ & 1 & 0.75 & 0.16 & 0.09 \\
$3 / 2 \mathrm{MDE}$ & 1 & 1.00 & 0.00 & 0.00 \\
$2 / 3 \mathrm{MDE}$ & 2 & 0.35 & 0.38 & 0.27 \\
$1 \mathrm{MDE}$ & 2 & 0.86 & 0.09 & 0.05 \\
$3 / 2 \mathrm{MDE}$ & 2 & 1.00 & 0.00 & 0.00 \\
\hline
\end{tabular}




$$
\left.\mathbf{x}_{t}=\underset{\mathbf{x}_{1}-\mathrm{x}_{0}}{\mathbf{A}}{ }_{\mathrm{x}_{\mathrm{t}}-\mathrm{x}_{0}}^{\mathbf{A}}\left(t-t_{0}\right)\right] \mathbf{k}+\mathbf{v},
$$

and the normal equation component for the $i$ th such block is

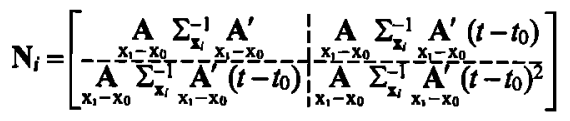

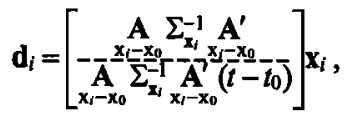

the solution of which is

$$
\Sigma_{\mathbf{k}}=\left(\sum_{i=1}^{n} \mathbf{N}_{i}\right)^{-1} \quad \mathbf{k}=\Sigma_{\mathbf{k}} \sum_{i=1}^{n} \mathbf{d}_{i}
$$

Because the 3-D orientation and hence the 3-D orientation rate of the kinematic network solution were loosely constrained, we introduced minimal a priori constraints of these TRS parameters. We used an a priori kinematic solution $\mathbf{X}_{\mathrm{AP}}$ to provide the coordinates used in the constraint function $\mathbf{C}$, which in this case consists of rows 1-3 and 8-10 of $\mathrm{Bv}_{\mathrm{v}}$ in (A7). The additional a priori normal equation component is

$$
\mathbf{N}_{\mathrm{AP}}=\left[\underset{\mathbf{k} A P-\mathbf{k}}{\mathbf{A}} \mathbf{B} \Sigma_{\mathbf{h}}^{-1} \mathbf{B}^{\prime} \underset{\mathbf{k}_{\mathrm{AP}}-\mathbf{k}}{\mathbf{A}^{\prime}}\right] \quad \mathbf{d}_{\mathrm{AP}}=\mathbf{0},
$$

where $\Sigma_{\mathbf{h}}$ is diagonal, containing the required variances of the six constrained orientation parameters. This a priori block could also be used to constrain the kinematic network scale rate to zero.

\subsection{Estimation of Epoch and Kinematic Datum Transformations}

Strictly speaking, a datum transformation is a function that changes the external geometry of a network coordinate set, leaving all estimable quantities unchanged. For epoch networks, subsets of the seven parameters (A2) are included; for kinematic networks, subsets of the 14 parameters (A5) are included. To estimate such a transformation between network solutions $\mathbf{x}$ and $y$ parameterized like (A1) or (A4), the observation model is

$$
\mathbf{y}+\mathbf{v}=\mathbf{t}+s \mathbf{R x}
$$

where $\mathbf{t}$ is a translation vector, $\mathbf{R}$ is a rotation matrix, $s$ is a scale parameter or vector, and $\mathbf{v}$ is a vector of Helmert residuals parameterized as for $\mathbf{x}$ and $\mathbf{y}$. All the vectors in (17) are partitioned into coordinate triplets (or sextuplets for kinematic networks), and the rotation matrix is a triplet (sextuplet) block diagonal. The model is linearized assuming that the parameters are small (this method is only useful for determining small reference frame differences) giving the Jacobean matrix (A3) for epoch networks or (A6) for kinematic networks. The least squares solution is iterative from a first guess $h_{0}$ (usually $\mathbf{h}_{0}=\mathbf{0}$ ), converging in $j$ iterations:

$$
\begin{gathered}
\Delta \mathbf{h}_{i}=\left(\mathbf{B}_{i}^{\prime} \mathbf{W} \mathbf{B}_{i}\right)^{-1} \mathbf{B}_{i}^{\prime} \mathbf{W}\left(\mathbf{y}-\overline{\mathbf{x}}_{i}\right), \\
\hat{\mathbf{h}}=\mathbf{h}_{0}+\sum_{i=1}^{j} \Delta \mathbf{h}_{i} \quad \Sigma_{\hat{\mathbf{b}}}=\left(\mathbf{B}_{j}^{\prime} \mathbf{W} \mathbf{B}_{j}\right)^{-1}, \\
\hat{\mathbf{v}}=\overline{\mathbf{x}}_{j}-\mathbf{y} \quad \Sigma_{\hat{\mathbf{v}}}=\Sigma_{(\mathbf{y}-\mathbf{x})}-\mathbf{B}_{j} \Sigma_{\hat{\mathbf{h}}} \mathbf{B}_{j}^{\prime} .
\end{gathered}
$$

The covariance matrix of observations in this least squares process is one of the following possibilities:

$$
\begin{gathered}
\Sigma_{(y-x)}=\Sigma_{y}+\Sigma_{x}, \\
\Sigma_{(y-x)}=\Sigma_{x}-\Sigma_{y}, \\
\Sigma_{(y-x)}=\Sigma_{x},
\end{gathered}
$$

where in (21a) $\mathbf{x}$ and $\mathbf{y}$ are independent solutions, in (21b) $\mathbf{y}$ is a least squares combination of solutions including $\mathbf{x}$, and in (21c) $\mathbf{y}$ is considered a correct reference network (Cross [1983] gives derivations of these equations). The first two cases are both used in this work, (1) for comparing A networks and R networks, and (2) for comparing the $G$ network with A networks.

\subsection{Estimation of Tectonic Plate Euler Vectors}

Rigid tectonic plate motion can be described by Euler vectors which are simply angular velocities. From the correlated station velocities of the kinematic solution we estimated absolute Euler vectors for seven major tectonic plates by ascribing most stations in the kinematic reference frame solution to one of these tectonic plates and discarding the remainder. Under the absolute Euler vector model the velocity vector $\mathbf{v}_{i}$ of each station $i$ (of $m$ stations) on plate $\mathrm{A}$ (of $p$ plates) with known geocentric position is described by a geocentric rotation about the 3-D Euler vector

$$
\Omega_{\mathrm{A}}=\left[\begin{array}{lll}
X_{\Omega_{\mathrm{A}}} & Y_{\Omega_{\mathrm{B}}} & Z_{\Omega_{\mathrm{C}}}
\end{array}\right]
$$

plus a residual velocity $v_{1}$, which gives a linearized observation model of the form $v=D \Omega+v$. This equation is solved by least squares in the usual way.

The absolute Euler vectors are correlated because the velocity parameters are correlated. This must be taken into account when deriving relative Euler vectors from pairs of absolute vectors. Relative Euler vectors are independent of a priori constraints on the kinematic TRS orientation rate. A relative Euler vector between two plates $A$ and $B$ is defined as ${ }_{A} \Omega_{B}=-B \Omega_{A}=\Omega_{B}-\Omega_{A}$. To better visualize Euler vectors, we express the Cartesian vector components in terms of latitude, longitude, and rotation rate (obtained by the usual geodetic formulas). The horizontal error ellipse and standard error of the rotation rate are extracted from the triplet covariance matrix of each Euler vector.

\section{Testing and Results}

\subsection{Input Data}

We use the first 18 months of weekly SINEX networks made available in the IGS densification pilot project with at least four IGS Analysis Centers participating; that is, August 20 1995, to February 221997 (GPS weeks 815-893). The time series graphs use GPS week as the time unit. See Beutler and Brockmann [1993], Kouba [1993], Zumberge et al. [1995, 1996, and 1997] for individual descriptions of the work of these agencies. The agencies are referred to by their three-character code names (Table 2). Weekly A network SINEX files from COD, EMR, GFZ and JPL were available from the start of the series. SIO 15 included from November 1, 1995 (GPS week 0825) and ESA is included from December 28, 1995 (GPS week 0839). The National Geodetic Survey began producing weekly SINEX files in GPS week 0898, and although their solutions are not used here, they have since been included in Newcastle's operational analysis. Figure 2 shows the number of stations estimated in the A networks during the period studied here. 


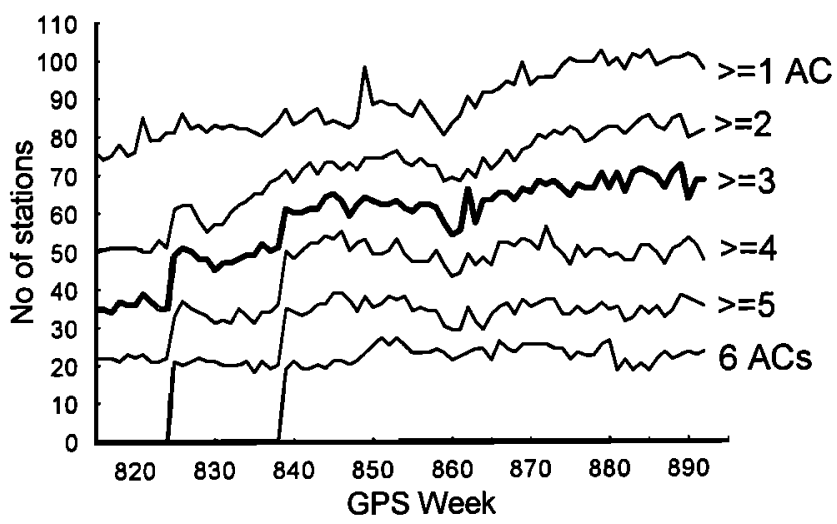

Figure 2. Number of stations appearing in $n$ A networks, for $n$ from $>=1$ to $>=6$, over the period of the experiment. Here $n>=3$ is the defining requirement for a global station, so the bold curve shows the number of stations in the $G$ network in each week. The steps in weeks 0825 and 0839 are the introduction of ACs SIO and ESA.

Weekly A network SINEX files from all these ACs were obtained from the IGS global data center, the Crustal Dynamics Data Information Service (see http://cddisa.gsfc.nasa.gov/) at NASA Goddard Space Flight Center. Each SINEX file was processed by matching each station against a SINEX format station catalogue that contains details of all IGS stations, including receiver and antenna types and antenna heights. Any discrepancies between each input SINEX file and the catalogue were automatically reported. The catalogue also provides unique parameter reference numbers for each station by means of which common station subsets in different solutions are known.

$\mathrm{R}$ networks are included in the polyhedra from the start of RNAAC involvement on July 11996 (GPS week 860), until the end of the series. Weekly RNAAC SINEX files from EUR, GSI, SIR and PGC were obtained from the CDDIS.

Of the RNAACs, we concentrate on EUR and GSI which each submitted a full series of problem-free weekly solutions over this period. Therefore our results demonstrate principles using pilot project data; they are not a definitive analysis.

\subsection{G network Estimation}

Figure 3 shows the variance components of the ACs as they evolved over the 18-month time series under the damped variance component estimation approach (11), with $d=0.2$ to allow long-term changes in AC scale factors but to prevent rapid fluctuations. With the exception of GFZ the components in Figure 4 show well-behaved slow evolution over time. The covariance matrix scaling of GFZ appears to be in increasingly worse agreement with its $G$ network residuals from week 0840 onward. Residual statistics showed that this was due to a changing scaling of the A network covariance matrix for an unknown reason, not to any deterioration in the quality of the GFZ network solution. We also tested fixed AC scale factors and found a marginal improvement in station repeatability using evolving scale factors.

Figure 4 shows the number of $A$ network station estimates from each $\mathrm{AC}$ deleted each week by the iterative data-snooping procedure (A9) with (12) operating at the $99.9 \%$ confidence level. The dotted curve indicates $5 \%$ of the total number of $\mathrm{A}$ network station estimates (which are the observations of the $G$ network estimation), showing that typically $2-3 \%$ of input data was discarded by the data snooping process, occasionally rising to $5-6 \%$. The reasons for the trends present in Figures 3 and 4 are unknown. Figure 5 shows the number of weeks in the series that each station was rejected from at least one A network (ordered from least to most rejections). The stations with many rejections are generally those with many antenna height blunders in AC A-SINEX files.

\subsection{Precision Assessment of Network Time Series}

Before looking at the time series results relative to kinematic solutions, we show a very simple and intuitive measure to assess the precision of the $A, R, G$, and $P$ networks in Figure 6. Each plot shows for each pair $f$ stations the RMS of the difference in baseline length between consecutive weekly estimates across the

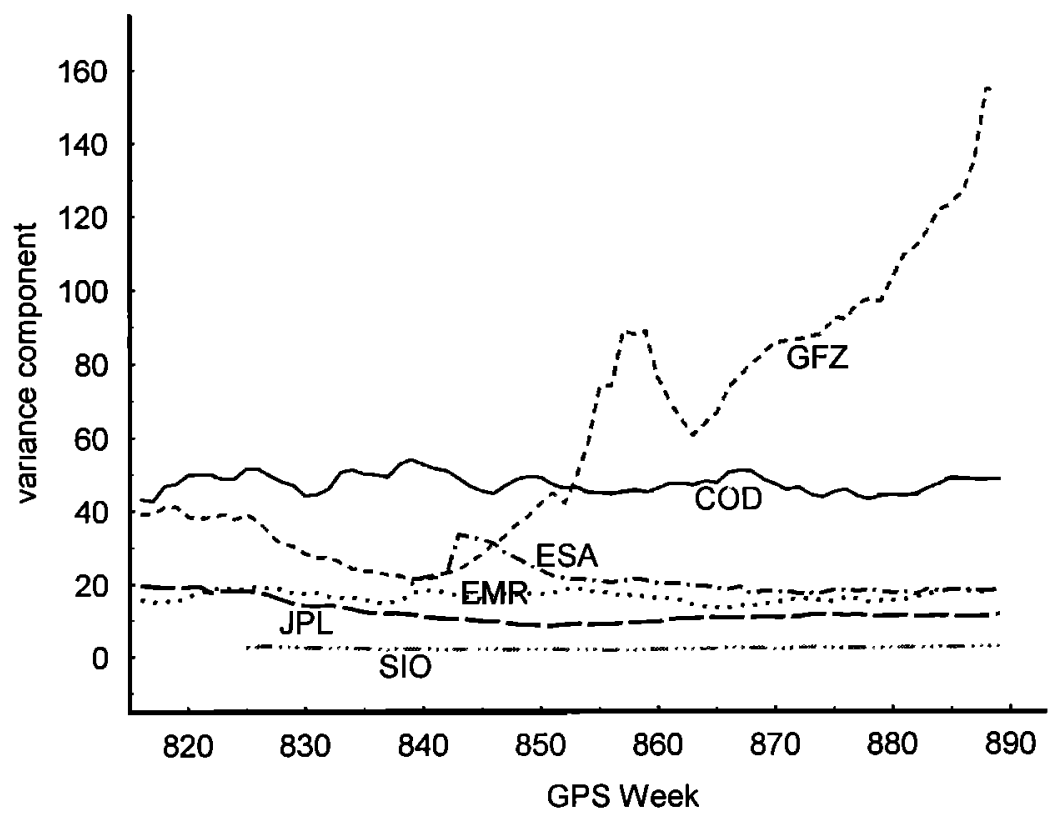

Figure 3. Evolving Analysis Center variance components under damped variance component estimation. 


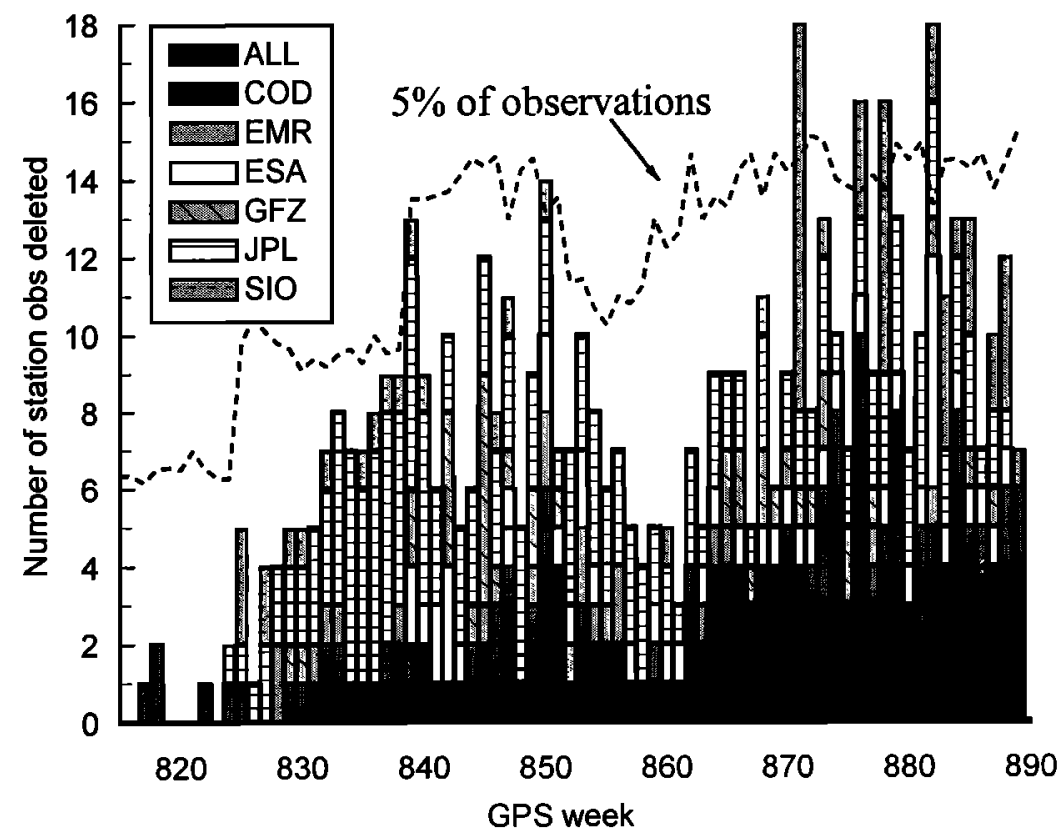

Figure 4. Number of A network station estimates iteratively rejected each week by multiparameter data snooping.

whole time series, plotted against baseline length. Considering only baseline lengths and only pairs of consecutive weeks is a simple way of removing artifacts of reference system definition and tectonic motion. The trail of dots on the outskirts of the $P$ network plot in Figure 6 is due to the noisy baselines between regional stations in different $\mathbf{R}$ networks.

\subsection{Kinematic Comparison of Networks}

If the $\mathrm{G}$ network methodology we have described is useful and the claims of high reliability are justified, we should be able to show that the quality of the $G$ network is superior to that of any A network in terms of long-term station position estimate repeatability. This is hinted at by Figure 6 , but our main demonstration uses kinematic residual series (as used by, e.g., van Dam et al. [1994] and Argus and Heflin [1995]). Kinematic residuals are just a way of removing the effects of TRS

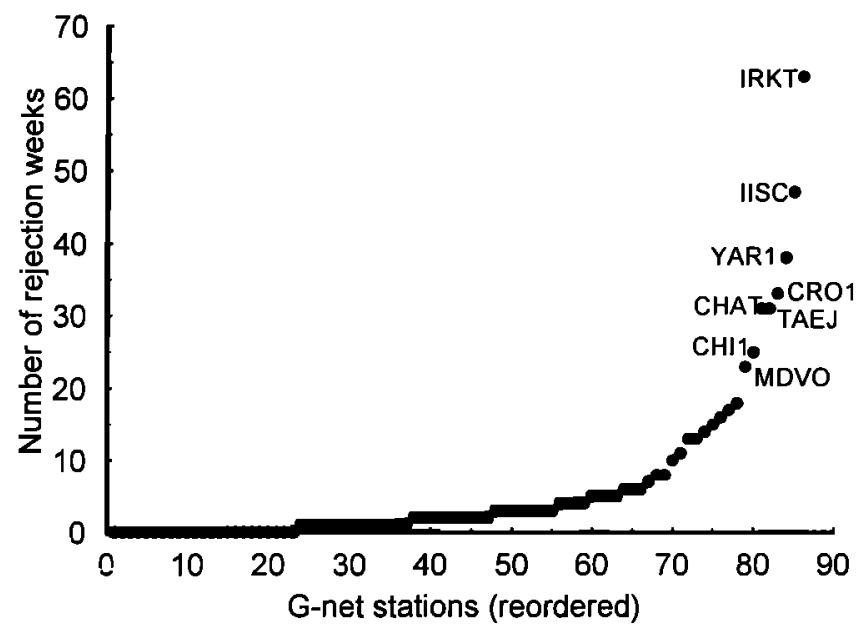

Figure 5. Number of weeks in series $0815-0889$ from which each global station was rejected from one or all A networks. The most frequently failing stations are named; these usually had various antenna height values among the ACs. differences and tectonic motion in order to compare time series of station position solutions.

We estimated a separate kinematic solution from each 18month time series of loosely constrained A networks and the $G$ network. Only those stations appearing in at least 20 members of the epoch solution series were included in the kinematic estimation. Exactly the same procedure was used to obtain the various A networks' and the G network's kinematic solution, and no outlier removal or solution iteration was performed. For each network series, seven-parameter Helmert transformations were estimated between each epoch network, and the kinematic solution was mapped to that epoch to obtain kinematic residual series for each A network and G network. These were expressed in local vertical, north, and east coordinates for each station. Owing to lack of space we do not show the kinematic residual series of individual stations. We show summaries of these series using different RMS statistics in Figure 7, Table 5, and Figure 8.

Figure 7 summarizes the time series of weekly RMS kinematic residual for each $A$ network and the $G$ network in terms of horizontal and vertical components. The $G$ network is clearly superior to any A network, most of which suffer from spikes in the RMS kınematic residuals. Although the $\mathrm{G}$ network is simply a combination of the A networks, the outlier detection and removal method is effective in removing these spikes.

Table 5 shows the RMS of the Helmert transformation translation (second through fifth columns) and scale (sixth column) parameters across the time series with respect to the mapped kinematic solution for each $A$ network and the $G$ network. This is a measure of the repeatability of the geocenter and network scale measurements. The $G$ network is equal to the best A network on the RMS 3-D translation parameter and is considerably better than any A network on the RMS scale parameter. The severnth and eighth columns of Table 5 present the summary RMS of the time series in Figure 7.

We computed the RMS of kinematic residuals for each station in each A network series. Figure 8 shows ordered plots of the station-by-station RMS kinematic residual statistic in the vertical, north, and east components for the six A network series and the 

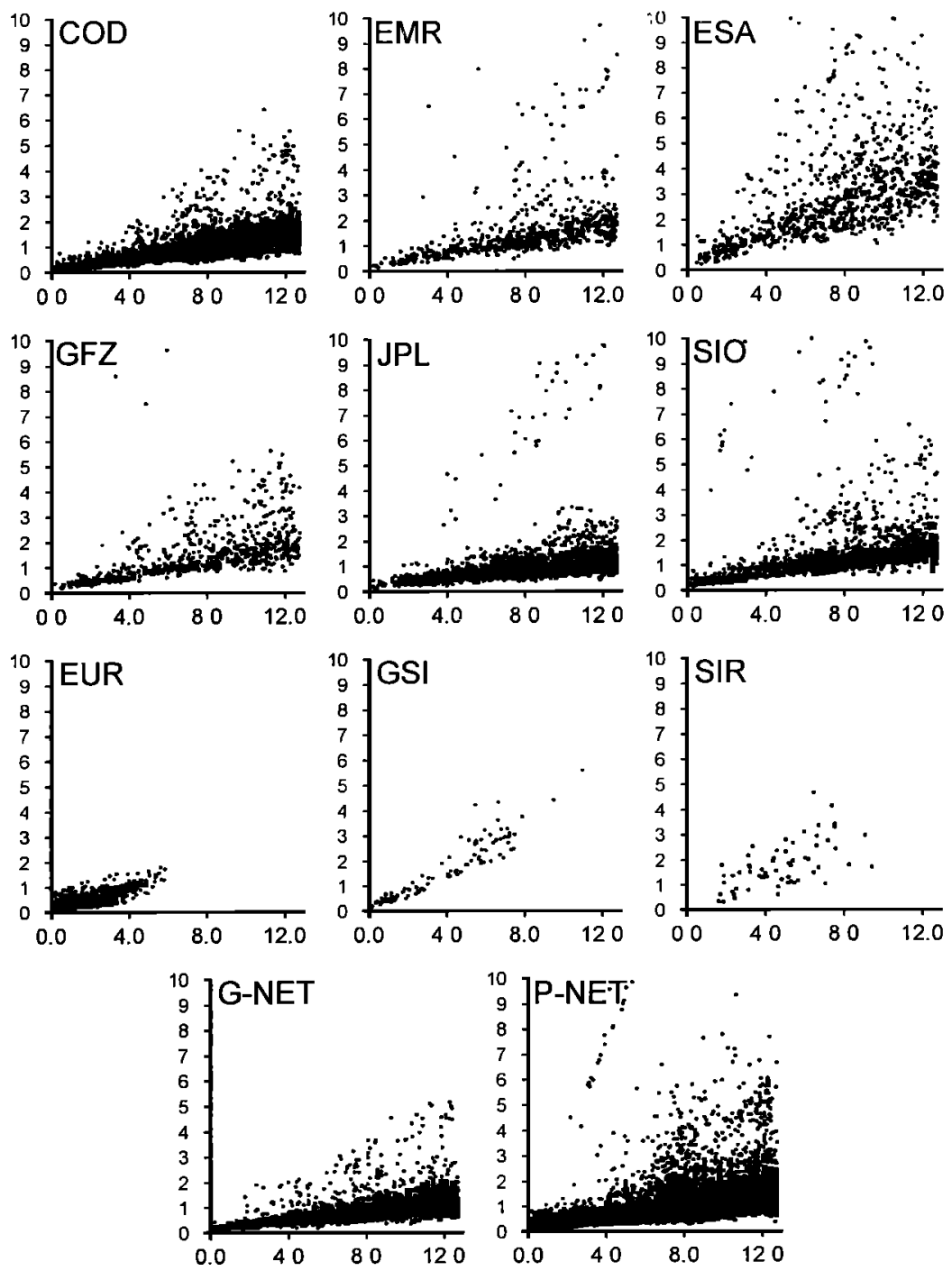

Figure 6. The $\mathrm{Y}$ axes represent baseline repeatability (centimetres), and $\mathrm{X}$ axes represent baseline length (thousands of kilometres). The plots show simple baseline repeatability in six A network series, three $\mathrm{R}$ network series, and the $G$ network and $P$ network series over 18 months. For each pair of stations, RMS discrepancy between pairs of consecutive weeks is plotted against distance between stations.

$\mathrm{G}$ network over 18 months. By arranging the stations in ascending order of the statistic and using "station set percentile" as the horizontal axis, we see comparative cumulative distributions of this statistic for each A network and the $G$ network. The RMS residual of the median station is $7.2 \mathrm{~mm}$ in height, $2.4 \mathrm{~mm}$ in latitude, and $3.0 \mathrm{~mm}$ in longitude.

It is clear from Figure 8 that on this statistic the $G$ network outperforms the best A network. Even though the $G$ network is simply a combination of these six A networks, it is demonstrably more precise than any of them. This shows that bringing the various A networks together in a combined $G$ network and using variance component estimation and outlier detection methods in the $G$ network estimation is useful. This is our most important result in favor of the IGS distributed polyhedron assembly scheme.

\subsection{A Kinematic Polyhedron Solution}

We estimated a kinematic solution (station positions at a reference epoch plus constant station velocities) from the 18month $\mathrm{P}$ network time series: we called this the $\mathrm{kP}$ network.
Stations appearing in less than five weekly $\mathrm{P}$ networks were discarded. We do not show maps of station velocity vectors. Figure 9 shows an ordered plot of vertical station velocities with three standard deviation error bars in three categories: global stations appearing in at least $50 \mathrm{P}$ networks over the 18-month period, regional stations of RNAAC EUR, and regional stations of RNAAC GSI. The stations in the second two categories have a maximum time series length of 33 weeks. Since all station vertical velocities are expected to be within $\pm 10 \mathrm{~mm} \mathrm{yr}^{-1}$, this plot shows the limitations of the short time series used here for vertical analyses.

We went on to estimate absolute Euler rotation vectors of seven major tectonic plates using the $\mathrm{kP}$ network station velocities as input data. We also applied the same method to the IGS stations listed in ITRF94 (using ITRF94 velocities) to obtain an Euler vector for each plate. Locations of ITRF94 stations are given by Boucher et al. [1996]. The kP network was aligned with ITRF94 by estimating and applying a 14-parameter datum transformation. This is required to allow direct comparison of the kP network with ITRF94 since absolute Euler vectors are not 


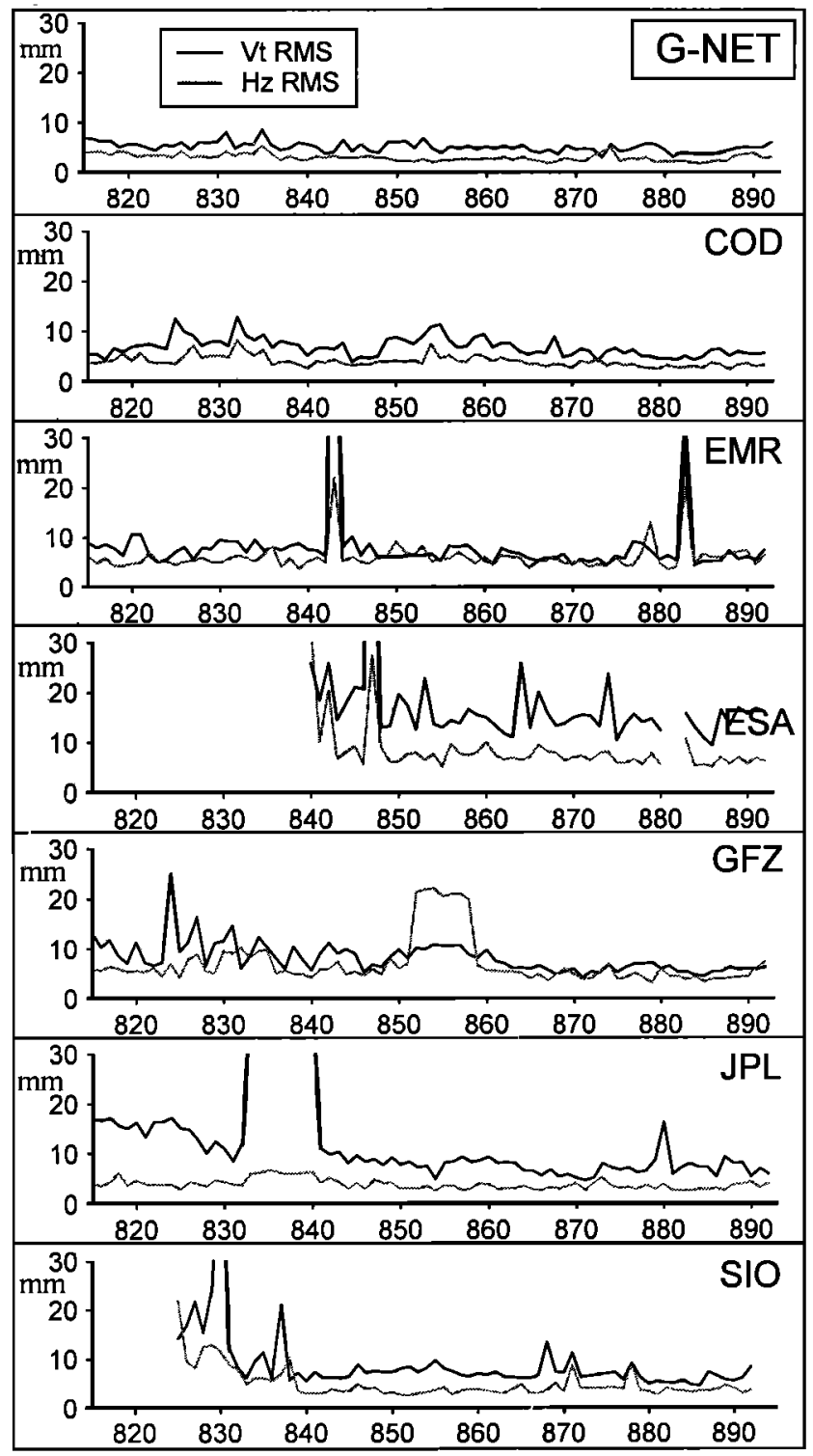

Figure 7. The $\mathrm{Y}$ axes represent vertical and horizontal kinematic residual RMS (millimetres) for each weekly A network and the $G$ network, and $\mathrm{X}$ axes represent Global Positioning System week. estimable quantities (without adopting some arbitrary kinematic datum, which we have carefully avoided). Table 6 gives the stations used on each plate, the RMS horizontal station velocity before fitting the Euler vectors, and the RMS residual velocity after fitting the vector. This was done separately for our $\mathrm{kP}$ network velocities and for the ITRF94 velocities of the IGS stations listed in ITRF94. Because the Eurasian and North American plates are much more densely sampled than the others in the $\mathrm{kP}$ network, we used only a fraction of the $\mathrm{kP}$ network stations available for those two plates. For this simple analysis we assumed the Australian and Indian plates to be one rigid plate.

We then looked at relative Euler vectors between pairs of the seven plates. We compared the relative Euler vector estimates of the kP network, ITRF94 GPS subset, and the NUVEL-1A geological model (DeMets et al. [1994] as quoted by McCarthy [1996]). Table 7 shows the magnitudes of vector differences between pairs of these three estimates for each pair of plates. The vector differences between the ITRF94 GPS subset and NUVEL$1 \mathrm{~A}$ are in the range $0.042-0.224^{\circ} \mathrm{Ma}^{-1}$, with an average of $0.115^{\circ}$ $\mathrm{Ma}^{-1}$. The differences between the 18 -month $\mathrm{kP}$ network and NUVEL-1 $\mathrm{A}$ are in the range $0.010-0.214^{\circ} \mathrm{Ma}^{-1}$, with an average of $0.111^{\circ} \mathrm{Ma}^{-1}$. The differences between the 18-month $\mathrm{kP}$ network and ITRF94 GPS subset are in the range 0.028-0.194 $\mathrm{Ma}^{-1}$, with an average of $0.118^{\circ} \mathrm{Ma}^{-1}$. This shows that our combined network, using only 18 months of data, is in agreement with the plate motions given by both ITRF94 and NUVEL-1A at the level of the difference between those two models. Interestingly, the equivalent values for the older NUVEL-1 model [McCarthy, 1992] are $0.142^{\circ} \mathrm{Ma}^{-1}$ between ITRF94 and NUVEL-1; and $0.165^{\circ} \mathrm{Ma}^{-1}$ between $\mathrm{kP}$ network and NUVEL-1. This is clear evidence that the geomagnetic reversal timescale adjustment [DeMets et al., 1994] made between the two versions of the NUVEL model has brought it into better agreement with both the geodetic models examined here. The best agreement between the three sets of Euler pole estımates is for the EurasiaNorth America pole, which is as we would expect since these two continents carry far more first-order geodetic stations than do other parts of the crust.

\subsection{Regional Networks: Attachment or Combination?}

To test the $\mathrm{R}$ network attachment method, we implemented an alternative combination method of all $A$ networks and $R$

Table 5. Repeatability of Weekly Helmert Parameters and Residuals

\begin{tabular}{lcccccccc}
\hline & \multicolumn{3}{c}{ RMS of Weekly Helmert Parameters } & & \multicolumn{2}{c}{ RMS Residuals } \\
\cline { 2 - 5 } \cline { 8 - 9 } & X, $\mathrm{mm}$ & $\mathrm{Y}, \mathrm{mm}$ & $\mathrm{Z}, \mathrm{mm}$ & $3 \mathrm{D}^{\mathrm{a}}, \mathrm{mm}$ & Scale, ppb & & Vertical, mm & Horizontal, mm \\
\hline G Net & 8.1 & 11.4 & 22.0 & 26.0 & 0.23 & & 5.2 & 3.0 \\
COD & 7.4 & 9.6 & 23.0 & 26.0 & 0.40 & & 7.0 & 4.1 \\
EMR & 19.2 & 18.9 & 75.3 & 80.0 & 0.74 & & 10.5 & 6.6 \\
ESA & 16.5 & 26.4 & 59.3 & 61.8 & 1.55 & & 18.2 & 9.8 \\
GFZ & 24.9 & 40.4 & 56.3 & 73.6 & 0.45 & & 8.7 & 8.4 \\
JPL & 8.2 & 10.4 & 30.4 & 33.2 & 0.94 & & 15.8 & 3.9 \\
SIO & 10.5 & 32.0 & 45.9 & 56.9 & 0.48 & & 11.1 & 5.9 \\
\hline
\end{tabular}

Helmert transformation parameters are estımated between each weekly epoch solution and the mapped kınematıc solution

${ }^{\mathrm{a}}$ Three-dimensional vector length of $\mathrm{X}, \mathrm{Y}$, and $\mathrm{Z}$ parameters. 


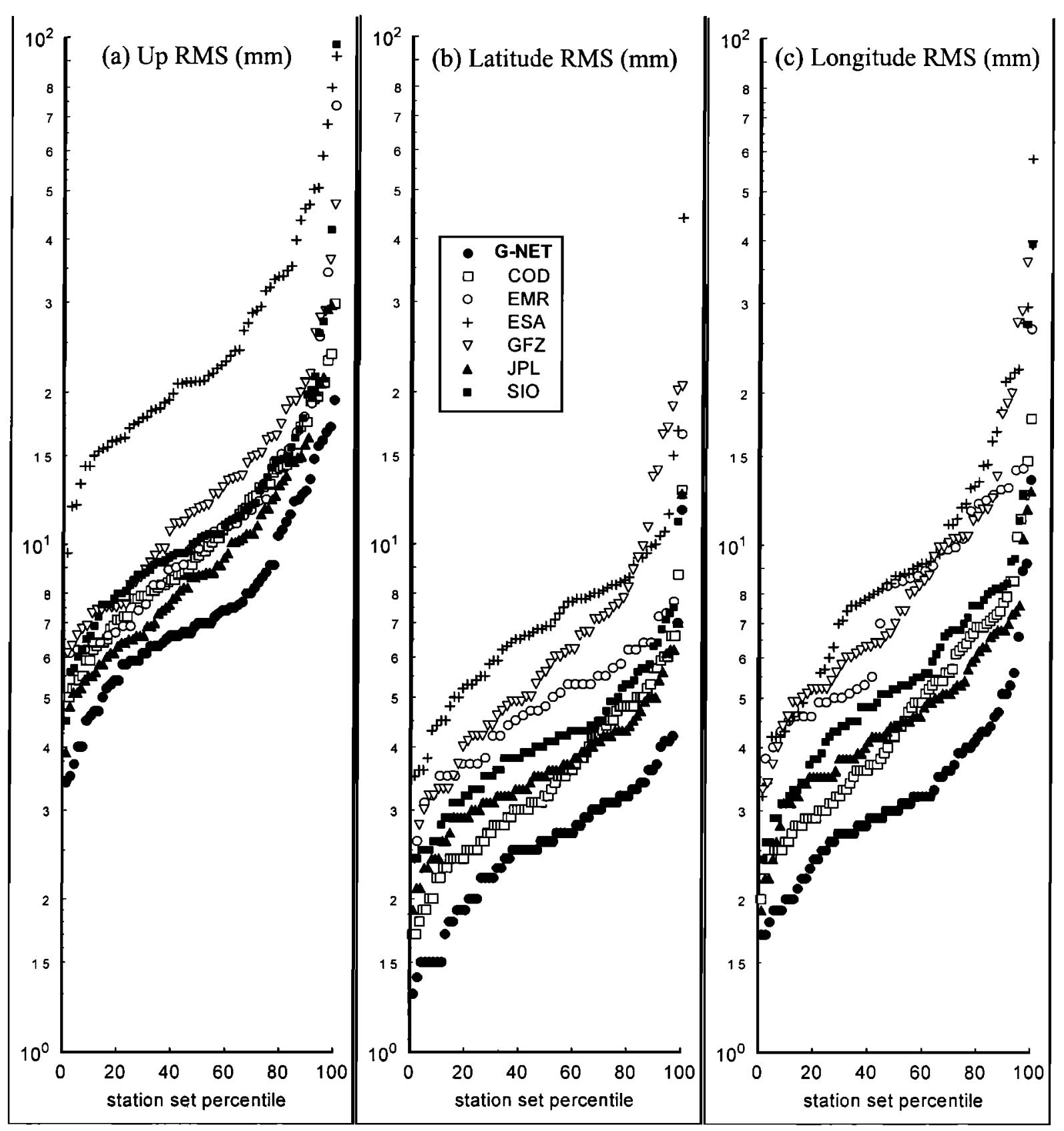

Figure 8. Ordered logarithmic plots of RMS kinematic residual for each station in each tıme series solution in (a) height, (b) latitude, and (c) longitude. The stations are not identified but are arranged in ascending order of RMS kinematic residual so the cumulative distribution of station RMS kinematıc residuals can clearly be seen for each contributing A network and our resulting G network. The median RMS residual for our G network is $7.2 \mathrm{~mm}$ in height, $2.4 \mathrm{~mm}$ in latitude, and $3.0 \mathrm{~mm}$ in longitude.

networks, using the series of evolving A network variance components previously determined in the $G$ network estimation and leaving $\mathrm{R}$ network covariance matrices unchanged. We excluded those station observations that were excluded in iterative data snooping in the $G$ network estimation. In Figure 10 we show only $\mathrm{R}$ network stations, with separate ordered series for the global junction stations and regional stations of the EUR (European) and GSI (Japanese) regional networks. This shows that regional station horizontal repeatability is improved by these networks being attached to the $G$ network rather than combined with it. This is especially true of the GSI R network. We also see that the repeatability of the global junction stations is slightly degraded by allowing them to be influenced by $R$ networks in the combination method.

\subsection{Are Scale and Geocenter Rate Differences Significant?}

Both network scale and geocenter are estimable quantities of the A, G, and P networks. We used the methods of Teunissen [1986] and Kosters and Kok [1989] to assess the statistical significance of the scale (at 1996.0), 3-D translation (at 1996.0), scale rate, and 3-D translation rate parameters between kinematic 


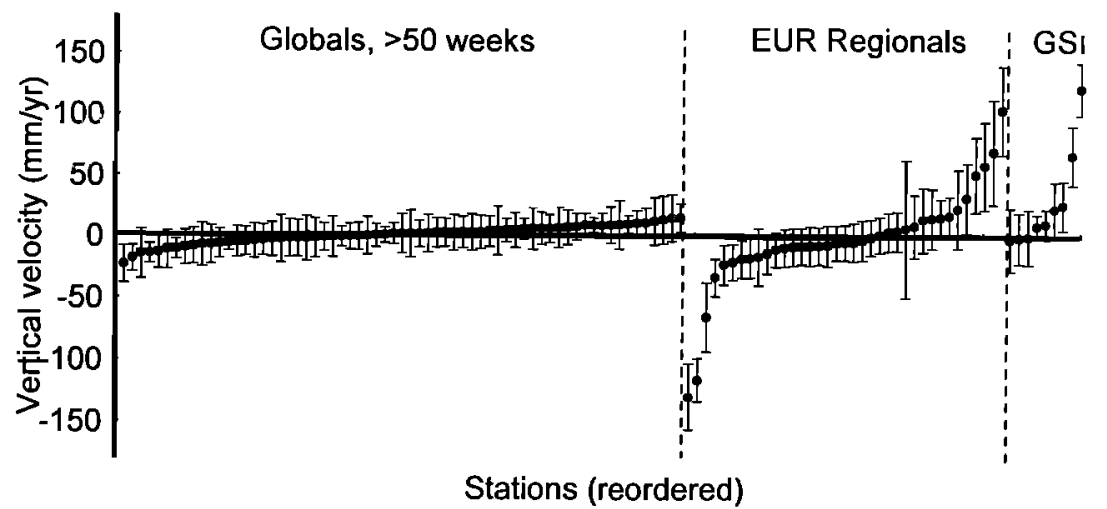

Figure 9. Ordered plots of station vertıcal velocity for three station subsets. (1) global stations occurring in at least 50 P networks, (2) EUR regionals, (3) GSI regionals. Error bars show three standard error confidence intervals.

A networks and the kinematic $G$ network and similarly between the kinematic P network and ITRF94 (Tables $8 \mathrm{a}$ and $8 \mathrm{~b}$ ). The statistical test uses the general hypothesis test (A9) with the appropriate rows of (A6) giving the $\mathbf{C}$ matrix. The conclusion is that there are statistically significant differences between $\mathrm{A}$ networks and the $G$ network in 3-D translation at 1996.0, network scale at 1996.0, and 3-D translation rate, but not in scale rate (except in one case). There are significant differences between the KP network and ITRF94 in 3-D translation at 1996.0, 3-D translation rate, and scale rate, but not in scale at 1996.0.

\section{Conclusions}

\subsection{Summary}

The weekly IGS polyhedron assembled by the GNAAC procedure developed here as part of the IGS distributed processing pilot project is superior to the global GPS network solution of any individual IGS Analysis Center. The polyhedron TRF is suitable for precise surveying and geodynamics applications requiring global precision, offering weekly reobservation and high worldwide GPS coverage. Compared to IGS AC weekly A networks, the polyhedron assembled here is more dense and inclusive, more precise (in terms of long-term repeatability of station positions and geocenter), more robust and reliable (in both statistical and organizational terms), and more easily extended, than any individual Analysis Center weekly solution. The IGS polyhedron assembled by distributed processing should therefore proceed as the standard weekly GPS terrestrial reference frame.

The ACs that achieve better time series repeatabilities of station and geocenter positions are in closer agreement with each other in each weekly solution than they are with the ACs of poor

Table 6. Statistics of Horizontal Velocities Residuals to Estimated Plate Motion Models

\begin{tabular}{|c|c|c|c|c|c|c|}
\hline \multirow[t]{2}{*}{ Plate } & \multicolumn{3}{|c|}{ kP Network Stations RMS Velocity } & \multicolumn{3}{|c|}{ ITRF94 IGS Stations RMS Velocity } \\
\hline & Number $^{b}$ & $\begin{array}{l}\text { Before Model, } \\
\qquad \mathrm{mm} \mathrm{yr}\end{array}$ & $\begin{array}{l}\text { After Model, } \\
\qquad \mathrm{mm} \mathrm{yr}^{-1}\end{array}$ & Number & $\begin{array}{l}\text { Before Model, } \\
\qquad \mathrm{mm} \mathrm{yr}\end{array}$ & $\begin{array}{l}\text { After Model, } \\
\qquad \mathrm{mm} \mathrm{yr}^{-1}\end{array}$ \\
\hline Africa $^{\mathrm{C}}$ & 4 & 154 & 5.5 & 2 & 13.2 & 0.0 \\
\hline Antarctic ${ }^{d}$ & 4 & 8.1 & 4.1 & 3 & 119 & 91 \\
\hline Australia ${ }^{\mathbf{e}}$ & 7 & 34.8 & 6.5 & 2 & 37.1 & 00 \\
\hline Eurasia $^{r}$ & 16 & 135 & 5.6 & 18 & 14.2 & 2.0 \\
\hline North America ${ }^{g}$ & 15 & 10.2 & 2.9 & 15 & 10.5 & 2.8 \\
\hline Pacific" & 11 & 35.7 & 11.6 & 3 & 30.9 & 6.1 \\
\hline South America' & 8 & 9.6 & 4.5 & 4 & 10.4 & 5.0 \\
\hline
\end{tabular}

'This is the subset of ITRF94 stations which are IGS GPS stations. All such stations were used (see Boucher et al. [1996] foi details).

bThis is the number of stations on each plate, each contributing two velocity components. The IGS fourcharacter station names of the stations used is given below for each plate.

'The $\mathrm{kP}$ network stations used are HART, MALI, MASP, and SEYI.

${ }^{\mathrm{d}}$ The $\mathrm{kP}$ network stations used are CASI, DAV1, KERG, and OHIG.

'The kP network stations used are AUCK, COCO, DGAR, HOB2, PERT, TIDB, and YARI.

'The kP network stations used are ANKR, BOR1, BRUS, KIRU, KIT3, KOSG, MADR, METS, NYAL, ONSA, POL2, POTS, SHAO, TAIW, TROM, and WTZR.

${ }^{8}$ The $k P$ network stations used are ALBH, ALGO, BRMU, DRAO, FAIR, GOLD, KELY, MDO1, NLIB, RCM5, REYK, STJO, THU1, WES2, and YELL.

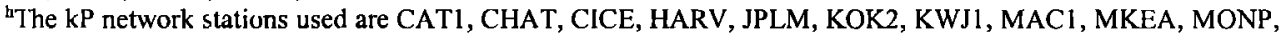
and PAMA.

'The kP network stations used are ASC1, AREQ, BOGT, BRAZ, FORT, KOUR, LPGS, and SANT. 
Table 7. Magnitude of Relative Euler Vector Differences Between Kinematic Solutions

\begin{tabular}{|c|c|c|c|c|c|c|c|}
\hline & Difference $^{a}$ & Antarctic & Australia & Eurasia & $\begin{array}{l}\text { North } \\
\text { America }\end{array}$ & Pacific & $\begin{array}{c}\text { South } \\
\text { Americ } \\
\text { a }\end{array}$ \\
\hline Africa & $\begin{array}{l}\text { kP-ITRF } \\
\text { ITRF- } \\
\text { NUVEL } \\
\text { kP-NUVEL }\end{array}$ & $\begin{array}{c}0.107 \\
0.096 \\
0.119\end{array}$ & $\begin{array}{c}0.164 \\
0.049 \\
0.124\end{array}$ & $\begin{array}{c}0.113 \\
0.091 \\
0.026\end{array}$ & $\begin{array}{c}0.101 \\
0.042 \\
0.059\end{array}$ & $\begin{array}{c}0.129 \\
0.158 \\
0.102\end{array}$ & $\begin{array}{c}0.100 \\
0.161 \\
0.105\end{array}$ \\
\hline Antarctic & $\begin{array}{l}\text { kP-ITRF } \\
\text { ITRF- } \\
\text { NUVEL } \\
\text { kP-NUVEL }\end{array}$ & & $\begin{array}{r}0.102 \\
0.098 \\
0.010\end{array}$ & $\begin{array}{r}0.089 \\
0.131 \\
0.104\end{array}$ & $\begin{array}{c}0.065 \\
0.097 \\
0.093\end{array}$ & $\begin{array}{c}0.194 \\
0.205 \\
0.109\end{array}$ & $\begin{array}{c}0.102 \\
0.149 \\
0.214\end{array}$ \\
\hline Australia & $\begin{array}{l}\text { kP-ITRF } \\
\text { ITRF- } \\
\text { NUVEL } \\
\text { kP-NUVEL }\end{array}$ & & & $\begin{array}{c}0.098 \\
0.050 \\
0.108\end{array}$ & $\begin{array}{c}0.103 \\
0.016 \\
0.093\end{array}$ & $\begin{array}{c}0.183 \\
0.167 \\
0.121\end{array}$ & $\begin{array}{c}0.173 \\
0.113 \\
0.219\end{array}$ \\
\hline Eurasia & $\begin{array}{l}\text { kP-ITRF } \\
\text { ITRF- } \\
\text { NUVEL } \\
\text { kP-NUVEL }\end{array}$ & & & & $\begin{array}{c}0.028 \\
0.050 \\
0.041\end{array}$ & $\begin{array}{c}0.136 \\
0.148 \\
0.109\end{array}$ & $\begin{array}{c}0.089 \\
0.091 \\
0.117\end{array}$ \\
\hline North America & $\begin{array}{c}\text { kP-ITRF } \\
\text { ITRF- } \\
\text { NUVEL } \\
\text { kP-NUVEL }\end{array}$ & & & & & $\begin{array}{c}0.150 \\
0.152 \\
0.133\end{array}$ & $\begin{array}{c}0.072 \\
0.121 \\
0.152\end{array}$ \\
\hline Pacific & $\begin{array}{l}\text { kP-ITRF } \\
\text { ITRF- } \\
\text { NUVEL } \\
\text { kP-NUVEL }\end{array}$ & & & & & & $\begin{array}{l}0.173 \\
0.224 \\
0.168\end{array}$ \\
\hline
\end{tabular}

All figures in the table are in units of degrees per million years.

${ }^{a}$ For each pair of plates the first row shows the difference between solutions ITRF94 and kP network; the second shows the difference between ITRF94 and NUVEL-1A; the third shows the difference between the kP network and NUVEL-1A.

repeatability. This is a conclusion of the results presented here which is not obvious, and it is perhaps the most far-reaching conclusion of this work. It indicates that the differences in A networks in a single week are not due to constant systematic differences in $\mathrm{AC}$ analysis (which could lead to the $\mathrm{AC}$ with best repeatability being consistently distant from the others) but rather behave like random errors. This leads to the A networks with high repeatability being close to the least squares combination of the A networks (i.e., the $G$ network) and hence achieving low estimated variance components and high influence over the $G$ network. That these variance components are physically meaningful is shown by the result that the $G$ network achieves

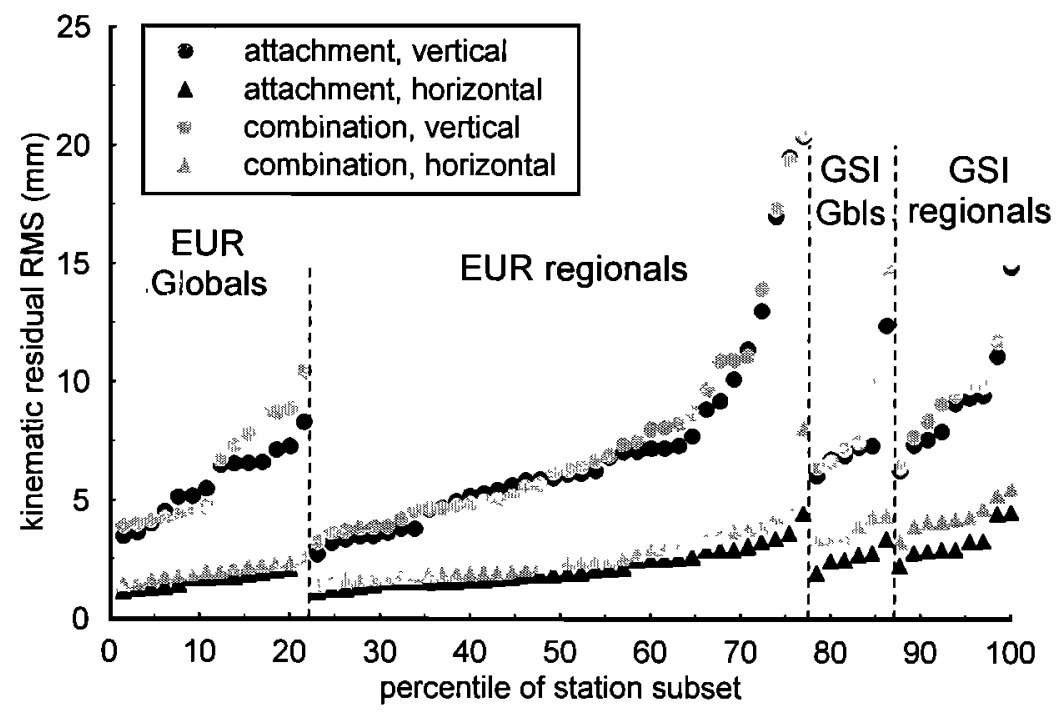

Figure 10. Ordered plots of kP network kinematic residual RMS for R network station time series. Vertical and horizontal components are shown separately. 
Table 8a. Significance of Epoch Transformation Parameters Between Kinematic Networks

\begin{tabular}{|c|c|c|c|c|c|c|c|c|}
\hline \multirow{3}{*}{ Code } & \multicolumn{5}{|c|}{ 'Thiee-Dimensional Translation at Epoch 1996.0} & \multicolumn{3}{|c|}{ Scale at Epoch 1996.0} \\
\hline & \multicolumn{3}{|c|}{ Parameters, mm } & \multicolumn{2}{|c|}{ Significance } & \multirow[t]{2}{*}{ Scale, ppb } & \multicolumn{2}{|c|}{ Significance } \\
\hline & $\mathrm{X}$ & $\mathrm{Y}$ & Z & $T$ & $95 \%{ }^{2}$ & & $T$ & $95 \%$ \\
\hline $\operatorname{COD}^{\mathrm{b}}$ & 18.6 & 21.9 & -9.5 & 473 & yes & 0.378 & 19.5 & yes \\
\hline $\mathrm{EMR}^{\mathrm{b}}$ & 36.2 & -113.6 & 18.9 & 224 & yes & -0.400 & 26.2 & yes \\
\hline $\mathrm{ESA}^{\mathrm{b}}$ & 9.7 & 72.1 & 12.7 & 901 & yes & 2.24 & 379 & yes \\
\hline GFZ $^{b}$ & -61.2 & -33.9 & 6.4 & 400 & yes & 0.171 & 11.1 & yes \\
\hline $\mathrm{JPL}^{\mathrm{b}}$ & 15.5 & 29.5 & 26.6 & 212 & yes & -0.421 & 121 & yes \\
\hline SIO $^{b}$ & 12.2 & 4.6 & 38.6 & 158 & yes & -0.504 & 172 & yes \\
\hline ITRF94" & -10.1 & -32.1 & 32.9 & 36.5 & yes & 0.581 & 0.976 & no \\
\hline
\end{tabular}

a'Yes' in this column indicates that the parameter is significantly different from zero at the $95 \%$ confidence level.

${ }^{b}$ Transformation is between the named kinematic A network and our kinematic $\mathrm{G}$ network.

'Transformation is between the 52 stations common to ITRF94 and our kP network.

superior long time series repeatability to any A network. This is not statistically preordained: improving long-term repeatability is not only a test of precision but is also a test of accuracy. The $G$ network results agree better than the A networks with the simplest kinematic model (that residual crustal motions after removing modeled effects, such as solid Earth tides, etc., are linear in time with little stochastic variation). Testing agreement with this model is therefore more than a statistical exercise; it demonstrates the potential for enhancing geophysical interpretation. This conclusion on its own justifies this work and the structure of the IGS densification project.

A network station estimates have a typical overall redundancy of $78 \%$ in the $G$ network combination (that is, an average redundancy number per coordinate triplet of 2.3 out of 3 ). The average coordinate triplet redundancy numbers for particular A networks vary between 1.6 and 2.8 after variance component scaling. Multiplicative variance components can be determined on weekly A network components of the $\mathrm{G}$ network combination with a typical $90 \%$ confidence interval of $\pm 20 \%$. Typical vertical MDEs in the $G$ network combination are $30-70 \mathrm{~mm}$ but large values up to $450 \mathrm{~mm}$ occur for high redundancy, low-precision station observations. The alignment of the largest MDE at a GPS station (vertical) with the most common blunder type (antenna height error) is a weakness of design in GPS station installations which could conceivably be avoided.

On the basis of kinematic residual series and Helmert parameter series the $G$ network shows much better station position repeatability and network scale repeatability than any $A$ network and geocenter repeatability equal to the best A network. G network kinematic residual RMS at the median station is 7.2 $\mathrm{mm}$ in the vertical and $2-3 \mathrm{~mm}$ in the horizontal. The range of station kinematic residual RMS is $3.4-19.3 \mathrm{~mm}$ in the vertical and $1.4-13.5 \mathrm{~mm}$ in the horizontal. Centimeter-level precision can therefore be claimed for all stations with respect to the global frame, and millimeter-level precision can be claimed for the best stations.

There is weak evidence that the $\mathbf{R}$ network attachment method used gives superior time series repeatability to a combination method, and it is certainly to be preferred on theoretical grounds. The attachment method is preferred because it insulates the $G$

Table 8b. Significance of Transformation Rate Parameters Between Kinematic Networks

\begin{tabular}{|c|c|c|c|c|c|c|c|c|}
\hline \multirow{3}{*}{ Code } & \multicolumn{5}{|c|}{ Three-Dimensional Translation Rate } & \multicolumn{3}{|c|}{ Scale Rate } \\
\hline & \multicolumn{3}{|c|}{ Parameters, $\mathrm{mm} \mathrm{yr}^{-1}$} & \multicolumn{2}{|c|}{ Significance } & \multirow{2}{*}{$\begin{array}{c}\text { Scale, } p \text { pb } \\
y r^{-1}\end{array}$} & \multicolumn{2}{|c|}{ Significance } \\
\hline & $\mathrm{X}$ & $\mathbf{Y}$ & $\mathbf{Z}$ & $T$ & $95 \%{ }^{\mathrm{a}}$ & & $T$ & $95 \%$ \\
\hline $\mathrm{COD}^{\mathrm{b}}$ & -17.1 & -16.6 & 13.6 & 170 & yes & 0.198 & 2.11 & no \\
\hline EMR $^{b}$ & -41.5 & 11.2 & 77.9 & 78 & yes & 0.547 & 1.17 & no \\
\hline $\mathrm{ESA}^{\mathrm{b}}$ & -6.4 & -16.1 & -53.1 & 155 & yes & 2.03 & 49.2 & yes \\
\hline $\mathrm{GFZ}^{\mathbf{b}}$ & 70.8 & -98.1 & 4.2 & 512 & yes & 0.022 & 4.12 & no \\
\hline $\mathrm{JPL}^{\mathbf{b}}$ & -19.8 & -22.6 & -40.5 & 173 & yes & -0.084 & $6 \mathrm{E}-6$ & no \\
\hline $\mathrm{SIO}^{\mathrm{b}}$ & -16.6 & 9.3 & -19.6 & 93 & yes & 0.036 & 1.30 & no \\
\hline ITRF94' & 10.5 & 26.4 & -31.5 & 56 & yes & 0.411 & 9.94 & yes \\
\hline
\end{tabular}

Read $6 \mathrm{E}-6$ as $6 \times 10^{-6}$

a'Yes' in this column indicates that the parameter is significantly different from zero at the $95 \%$ confidence level.

'Transformation is between the named kinematic A network and our kinematic $G$ network.

'Transformation is between the 52 stations common to ITRF94 and our kP network. 
network from regional station groups. With regional networks attached, the kinematic polyhedron has a maximum station time series residual RMS of $27 \mathrm{~mm}$ vertical and $14 \mathrm{~mm}$ horizontal. The kinematic polyhedron shows significant translation, translation rate, and scale rate differences to ITRF94. The RMS agreement for the 52 stations common to the $P$ network and ITRF94 at 1996.0 after removing the 14-parameter TRS difference is $8.1 \mathrm{~mm}$ vertical and $4.5 \mathrm{~mm}$ horizontal for positions, $4.8 \mathrm{~mm}$ vertical and $3.1 \mathrm{~mm}$ horizontal for velocities. Kinematic $P$ network station velocities fit a seven-plate estimated Euler vector tectonic model with horizontal plate velocity residual RMS of $4.1-11.6 \mathrm{~mm} \mathrm{yr}^{-1}$. The error ellipses of the estimated absolute Euler poles include the ITRF94 Euler pole for five of the seven plates. The magnitude of the difference in relative Euler vectors between our solution and the NUVEL-1A geological model is $0.041-0.324^{\circ} \mathrm{Ma}^{-1}$ depending on the plate pair.

\subsection{Future Work}

The methodology described in this paper has since been applied to routine weekly IGS analysis since September 1995 and is continuing to incorporate stations from an ever-growing global network of IGS stations (currently approaching 200). The GNAAC has continued to operate at Newcastle since that time producing solutions.

This paper has demonstrated that the geodetic resolution resulting from these accumulating GNAAC solutions should be sufficient to rigorously test geological models such as NUVEL$1 \mathrm{~A}$ within the next few years. With increased spatial sampling we could test whether the rigid plate kinematic model is a viable concept to explain most of the data or whether more general kinematic models (e.g., spherical strain rate tensor models) will be more appropriate. These investigations will include study of the statistical character of kinematic solutions, which could bias the determination of station velocity. Ultimately, a more accurate and higher-resolution description of the kinematics will improve dynamic interpretation.

To fully realize a GPS solution, which unifies all distance scales, would require the incorporation of data from thousands of stations, including dense regional arrays, and data from epoch campaigns. Since our method involves partitioning, it can readily use campaign solutions and regional network solutions as inputs. We therefore suggest that this approach be used as part of a coordinated effort to produce a GPS global geodetic master solution to enable more powerful geophysical analyses through improved coverage, resolution, precision, reliability, adherence to standards, and uniformity of documentation. Such an activity would also serve to improve the archives for use by future generations of Earth scientists. This work has formed the basis for such an undertaking, which at the time of writing is being planned as a working group activity within the University NAVSTAR (Navigation System by Timing And Ranging) Consortium.

\section{Appendix}

\section{A1. Linear Mapping of Coordinate Triplets to Helmert Parameters}

The linear function $\mathbf{B}$ in $\Delta \mathbf{y}=\mathbf{B h}$ for a small change $\Delta \mathbf{y}$ in a vector of coordinate triplets

$$
\left.\mathbf{y}=\left[\begin{array}{lll}
x_{1} & y_{2} & z_{3}
\end{array}\right] \cdots\left[\begin{array}{lll}
x_{n} & y_{n} & z_{n}
\end{array}\right]\right]
$$

due to a vector of seven reference system parameters

$$
\mathbf{h}=\left[\begin{array}{lllllll}
r_{x} & r_{y} & r_{z} & s & t_{x} & t_{y} & t_{z}
\end{array}\right]
$$

(rotations $r$ and translations $t$ with respect to the three axes and a scale factor $s$ ) is

$\mathbf{B}_{\mathbf{X}}=\left[\begin{array}{ccc:ccc:c:ccc}0 & z_{1} & -y_{1} & 0 & z_{2} & -y_{2} & \cdots & 0 & z_{n} & -y_{n} \\ -z_{1} & 0 & x_{1} & -z_{2} & 0 & x_{2} & \ldots & -z_{n} & 0 & x_{n} \\ y_{1} & -x_{1} & 0 & y_{2} & -x_{2} & 0 & \cdots & y_{n} & -x_{n} & 0 \\ x_{1} & y_{1} & z_{1} & x_{2} & y_{2} & z_{2} & \ldots & x_{n} & y_{n} & z_{n} \\ 1 & 0 & 0 & 1 & 0 & 0 & \cdots & 1 & 0 & 0 \\ 0 & 1 & 0 & 0 & 1 & 0 & \cdots & 0 & 1 & 0 \\ 0 & 0 & 1 & 0 & 0 & 1 & \cdots & 0 & 0 & 1\end{array}\right]^{\prime}$

If vector $\mathbf{y}$ also includes station velocities

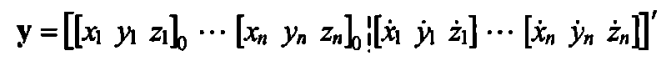

where the subscript 0 indicates that the position parameters refer to a certain arbitrary reference epoch, then $\mathbf{h}$ can include frame rate (time derivative) parameters:

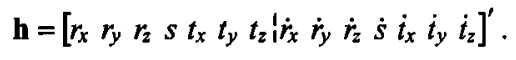

The Jacobean is then expanded to include the first derivative with respect to time of $\mathbf{B}_{\mathbf{x}}$, that is,

$$
\mathbf{B}=\left[\begin{array}{cc}
\mathbf{B}_{\mathrm{X}} & 0 \\
\mathbf{B}_{\mathrm{V}} & \mathbf{B}_{\mathrm{X}}
\end{array}\right],
$$

where

$$
\mathbf{B v}=\left[\begin{array}{ccc:ccc:c:ccc}
0 & \dot{z}_{1} & -\dot{y}_{1} & 0 & \dot{z}_{2} & -\dot{y}_{2} & \cdots & 0 & \dot{z}_{n} & -\dot{y}_{n} \\
-\dot{z}_{1} & 0 & \dot{x}_{1} & -\dot{z}_{2} & 0 & \dot{x}_{2} & \ldots & -\dot{z}_{n} & 0 & \dot{x}_{n} \\
\dot{y}_{1} & -\dot{x}_{1} & 0 & \dot{y}_{2} & -\dot{x}_{2} & 0 & \ldots & \dot{y}_{n} & -\dot{x}_{n} & 0 \\
\dot{x}_{1} & \dot{y}_{1} & \dot{z}_{1} & \dot{x}_{2} & \dot{y}_{2} & \dot{z}_{2} & \ldots & \dot{x}_{n} & \dot{y}_{n} & \dot{z}_{n} \\
0 & 0 & 0 & 0 & 0 & 0 & \ldots & 0 & 0 & 0 \\
0 & 0 & 0 & 0 & 0 & 0 & \ldots & 0 & 0 & 0 \\
0 & 0 & 0 & 0 & 0 & 0 & \ldots & 0 & 0 & 0
\end{array}\right]^{\prime}
$$

\section{A2. General Linear Hypothesis Testing}

Kosters and Kok [1989] discuss testing c-dimensional hypotheses (i.e., hypothesizing $c$ additional model parameters) against chi-square distributions where the null hypothesis is the usual LS model. The $(c \times c)$ reliability matrix $\mathbf{R}$ of the alternative hypothesis is:

$$
\mathbf{R}=\left(\mathbf{C}^{\prime} \Sigma_{\mathbf{x}}^{-1} \Sigma_{\mathbf{v}} \Sigma_{\mathbf{x}}^{-1} \mathbf{C}\right)^{-1} \lambda_{0},
$$

where $\mathbf{C}(m \times c)$ is the linear design matrix of the hypothesized model error in terms of the observations and $\lambda_{0}$ is the noncentrality parameter defined by $c$ together with the confidence level and power of the test (lookup tables for $\lambda_{0}$ are given by Baarda [1968]). The square roots of the eigenvalues of the reliability matrix and their eigenvectors are the magnitudes and directions, respectively, of the axes of the reliability hyperellipsoid, which contains the detectable error region. The hyperellipsoid surface is the marginally detectable error region. The chi-square test statistic is

$$
T=\hat{\mathbf{v}}^{\prime} \Sigma_{\mathbf{y}}^{-1} \operatorname{CRC}^{\prime} \Sigma_{\mathbf{y}}^{-1} \hat{\mathbf{v}} \sim \chi_{c}^{2} .
$$


Acknowledgments. This woik was funded in part by the Natural Environment Research Council under studentship 4/94/386/G (P. Davies) and under grant GR3/10041 (G. Blewitt). For all the data used in this paper, we are indebted to our collaborating institutions and colleagues of the International GPS Service, who are too numerous to mention here. We are particularly grateful for the guidance and leadership of Gerhard Beutler and the IGS Goveming Board for recognizing the potential for this research and for giving us the latitude to pursue it. A special thank you goes to Jan Kouba for his keen oversight and for his technical advice as IGS Analysis Center Coordinator.

\section{References}

Argus, D. F., and M. B. Heflin, Plate motion and crustal deformation estimated with geodetic data from the Global Positioning System, Geophys. Res. Lett., 22(15), 1973-1976, 1995.

Baarda, W., A testing procedure for use in geodetic networks, Netherlands Geodetic Commission, Publications on Geodesy New Series, 2(5), 1968

Beutler, G., and E. Brockmann (eds.), Proceedings of the IGS Workshop, Berne, 25-26 March 1993, Int. GPS. Serv. Cent. Bur., Pasadena, Calif., 1993.

Beutler, G., J. Kouba, and R. E. Neilan, Densification of the ITRF through regional GPS networks: organisational aspects, in Densification of the IERS Terrestrial Reference Frame through regional GPS networks, edited by J. F. Zumberge and R. Liu, pp. 6982, Int. GPS. Serv. Cent. Bur., Pasadena, Calif., 1995.

Blewitt, G., Advances in Global Positioning System technology for geodynamics investigations, in Contributions of Space Geodesy to Geodynamics: Technology, Geodyn. Ser., vol. 25, edited by D.E. Smith and D.L. Turcotte, pp. 195-213, AGU, Washington, D C, 1993.

Blewitt, G., GPS data processing methodology: From theory to applications, in GPS for Geodesy, edited by P.J.G. Teunissen and A. Kleusberg, pp 231-270, Springer-Verlag, New York, 1998.

Blewitt, G., M. B. Heflin, F. H. Webb, U. J. Lindqwister, and R. P. Malla, Global coordinates with centimeter accuracy in the international terrestrial reference frame using GPS, Geophys Res. Lett., 19(9), 853-856, 1992.

Blewitt, G., Y. Bock, and G Gendt, Regional clusters and distributed processing, in Proceedings of the IGS Analysis Center workshop. October 12th-14th 1993, Ottawa, Canada, edited by J. Kouba, pp. 62-91, Int. GPS. Serv. Cent. Bur., Pasadena, Calif, 1993a

Blewitt, G., M. B Heflin, K. J. Hurst, D. C. Jefferson, F. H. Webb, and J. F. Zumberge, Absolute far-field displacements from the 28 June 1992 Landers earthquake sequence, Nature, 361, 340-342, $1993 \mathrm{~b}$.

Blewitt, G., Y. Bock, and J. Kouba, Constructing the IGS Polyhedron by distributed processing, in Denstfication of the IERS Terrestrial Reference Frame through regional GPS networks, edited by J. F. Zumberge and R. Liu, pp. 21-38, Int. GPS Serv. Cent. Bur., Pasadena, Calif., 1995.

Bock. Y., et al., Detection of crustal deformation from the Landers earthquake sequence using continuous geodetic measurements, Nature, 36I, 337-340, 1993.

Boucher, C., and Z. Altamimi, Development of a conventional terrestria] reference frame, in Contributions of Space Geodesy to Geodynamics: Earth Dynamics, Geodyn. Ser., vol. 24, edited by D. E. Smith \& D L. Turcotte, pp. 89-97, AGU, Washington D. C., 1993.

Boucher, C., Z. Altamimı, M. Feissel, and P. Sillard, Results and analysis of the ITRF94, IERS Technical Note 20, Int. Earth Rotation Serv. Cent. Bur., Observ. de Paris, March 1996.

Cooper, M. A. R., Control surveys in civil engineering, Collins, London, 1987.

Cross, P.A., Advanced least squares applied to position fixing, N. E. London Polytech. Dept of Land Surv., Work. Pap. 6, London, 1983.

DeMets, C., R. Gordon, D. Argus, and S. Stein, Effect of recent revisions to the geomagnetic reversal timescale on estimates of current plate motions, Geophys. Rev. Lett., 2J(20), 2191-2194, 1994.

Grafarend, E. W., and B. Schaffrin, Vanance-covariance-component estimation of Helmert type, Surv. and Mapp. 39(3), 225-234, 1979.

Heflin, M. B., et al., Global geodesy using GPS without fiducial sites, Geophys. Res. Lett., 19(2), 131-134, 1992.
Herring, T. A., D. Dong, and R. W. King, Sub-milliarcsecond determination of pole position using Global Positioning System data, Geophys. Res. Lett., 18(10), 1893-1896, 1991.

Koch, K. R., Parameter estimation and hypothesis testing in linear models, Springer-Verlag, New York, 1987a.

Koch, K. R., Bayesian inference for variance components, Manuscr. Geod., 12, 309-313, $1987 \mathrm{~b}$.

Koch, K. R. Bayesian statistics for variance components with informative and noninformative priors, Manuscr. Geod., 13, 370-373, 1988.

Kosters, A. J. M., On the connection of geodetic pointfields in RETrig and related tests for model errors, Rep. Fac. of Geod., Math. Phys. Geod., 88.1, Delft Univ. of Technol., 1988.

Kosters, A. J. M., and J. J. Kok, Statistical testing and quality analysis of observations and transformation parameters in combining 3dimensional networks, paper presented at IAG Congress, Int. Assoc. of Geod., Edinburgh, Aug 3-12, 1989.

Kouba, J., (Ed.), Proceedings of the IGS Analysis Center workshop, October 12th-14th 1993, Ottawa, Canada, Int. GPS. Serv. Cent. Bur., Pasadena, Calif., 1993.

Larson K., J. Freymueller, and S. Philipsen, Global plate velocities from the Global Positioning System, J. Geophys. Res., 102(B5), 9961. 9982, 1997.

Lindqwister, U. J., A. P. Freedman, and G. Blewitt, Daily estimates of the Earth's pole position with the Global Positioning System, Geophys. Res. Lett., 19(9), 845-848, 1992.

McCarthy, D., IERS Standards, IERS Tech. Note 13, Int. Earth Rotation Serv. Cent. Bur., Observ. de Paris, 1992

McCarthy, D., IERS Conventions, IERS Tech. Note 21, Int. Earth Rotation Serv. Cent. Bur., Observ. de Paris, 1996

Mueller, I. I., Reference coordinate systems and frames: Concepts and realization, Bull. Geod., 59, 181-188, 1985.

Ou, Z., Approximative Bayes estimation for variance components, Manuscr. Geod., 16, 168-172, 1991.

Sahin, M., P. A. Cross, and P. C. Sellers, Variance component estimation applied to satellite laser ranging, Bull. Geod., 66, 284-295, 1992.

Segall, P. and J. L. Davis, GPS applications for geodynamics and earthquake studies, Annu. Rev. Earth Planet. Sci, 25, 301-336, 1997.

Teunissen, P. J. G., Adjusting and testing with the models of the affine and similarity transformation, Manuscr. Geod., 11, 214-225, 1986

van Dam, T. M., G. Blewitt, and M. B. Heflin, Atmospheric pressure loading effects on Global Positioning System coordinate determinations, J. Geophys. Res., 99(B12), 23,939-23,950, 1994.

Vigue, Y., S. M. Lichten, G. Blewitt, M. B. Heflin, and R. P. Malla, Precise determination of Earth's center of mass using measurements from the Global Positioning System, Geophys. Res Lett., 19(14), 1487-1490, 1992 .

Wolf, H., The Helmert block method - Its origin and development, in Proceedings of the Second International Symposium on Problems Related to the Redefinition of North American Geodetic Networks, pp. 319-326, U.S. Dep. of Commer., Washington, D. C., 1978.

Zumberge, J. F., and R. Liu (eds), IGS Workshop Proceedings: Densification of the IERS Terrestrial Reference Frame through regional GPS networks, Pasadena, California, November 30 . December 2, 1994, Int. GPS. Serv. Cent. Bur., Pasadena, Calif, 1995.

Zumberge, J. F., R. Liu, and R. Neılan (eds ), IGS Annual Report 1994, Int GPS Serv Cent Bur., Pasadena, Calif., 1995.

Zumberge J. F., M. P. Urban, R. Liu, and R. E. Neilan (Eds.), IGS Annual Report 1995, Int. GPS Serv. Cent. Bur., Pasadena, Calif., 1996.

Zumberge J. F., D. E. Fulton, and R. E. Neilan (Eds.), IGS Annual Report 1996, Int GPS Serv. Cent. Bur., Pasadena, Calif., 1997.

G. Blewitt, Nevada Bureau of Mines and Geology, and Seısmological Laboratory, University of Nevada, Reno, NV89557. (gblewitt@unr edu)

P. Davies, Ordnance Survey, Romsey Road, Southampton SO16 4GU, England, U K. (pdavies@ordsvy.gov.uk)

(Received June 3, 1999; revised November 22, 1999; accepted January 6, 2000.) 